IJMMS 28:6 (2001) 331-358

PII. S0161171201012017

http://ijmms.hindawi.com

(C) Hindawi Publishing Corp.

\title{
MULTIVARIABLE $q$-HAHN POLYNOMIALS AS COUPLING COEFFICIENTS FOR QUANTUM ALGEBRA REPRESENTATIONS
}

\author{
HJALMAR ROSENGREN
}

(Received 12 February 2001)

\begin{abstract}
We study coupling coefficients for a multiple tensor product of highest weight representations of the $\mathrm{SU}(1,1)$ quantum group. These are multivariable generalizations of the $q$-Hahn polynomials.
\end{abstract}

2000 Mathematics Subject Classification. 17B37, 33D70, 33D80.

1. Introduction. It is known that the Clebsch-Gordan coefficients for highest weight representations of $\mathrm{SU}(1,1)$ may be identified with three classical systems of orthogonal polynomials, namely, the Jacobi polynomials, the Hahn polynomials, and the continuous Hahn polynomials [41]. They correspond to the three conjugacy classes of one-parameter subgroups of $\mathrm{SU}(1,1)$. In our previous papers [29, 31] we found that many multi-variable polynomials occurring in the literature have a similar interpretation, as coupling coefficients for an $n$-fold tensor product of highest weight representations. This interpretation leads to simple proofs of many properties of such polynomials.

The purpose of this paper is to generalize some of these results to the context of quantum groups and $q$-series. Several authors have found that $q$-Hahn polynomials appear as Clebsch-Gordan coefficients for the $\mathrm{SU}(2)$ and $\mathrm{SU}(1,1)$ quantum groups [16, 19, 22, 34, 39]. More recently, Granovskiǐ and Zhedanov [11] and Koelink and Van der Jeugt [20] found similar interpretations of $q$-Racah polynomials and Askey-Wilson polynomials, respectively. In this paper, however, we only consider multivariable generalizations of $q$-Hahn polynomials. This case is more elementary and more similar to the Lie algebra case.

For comparison, we will summarize some of the basic facts of [29, 31]. Let, for $v>0$, $\mathscr{A}^{v}$ be the Hilbert space of analytic functions on the complex unit disc with norm

$$
\|f\|^{2}=\sum_{k=0}^{\infty} \frac{k !}{(v)_{k}}|\hat{f}(k)|^{2} .
$$

Here $f(z)=\sum \hat{f}(k) z^{k}$ and $(v)_{k}=v(v+1) \cdots(v+k-1)$ is the Pochhammer symbol. For $v>1$ one has the integral formula

$$
\|f\|^{2}=\frac{v-1}{\pi} \int_{|z|<1}|f(z)|^{2}\left(1-|z|^{2}\right)^{v-2} d x d y
$$


The norm of $\mathscr{A}^{v}$ is invariant under the transformations

$$
f(z) \longmapsto \frac{1}{(c z+d)^{v}} f\left(\frac{a z+b}{c z+d}\right), \quad\left(\begin{array}{ll}
a & b \\
c & d
\end{array}\right) \in \mathrm{SU}(1,1),
$$

which give rise to a unitary representation of a covering group of $\mathrm{SU}(1,1)$ on each space $\mathscr{A}^{v}$. This representation is irreducible and has the highest weight vector 1 of weight $-v$. The derived representation of the complexified Lie algebra $\mathfrak{s}[(2, \mathbb{C})$ is given by the densely defined operators

$$
X_{+}=-\frac{d}{d z}, \quad X_{-}=z^{2} \frac{d}{d z}+v z, \quad H=-\left(v+2 z \frac{d}{d z}\right),
$$

which satisfy the structure equations

$$
\left[X_{+} X_{-}\right]=H, \quad\left[H X_{+}\right]=2 X_{+}, \quad\left[H X_{-}\right]=-2 X_{-},
$$

and the $\mathfrak{s u}(1,1)$ reality conditions

$$
X_{ \pm}^{*}=-X_{\mp}, \quad H^{*}=H .
$$

Consider a Hilbert tensor product $\mathscr{A}^{v_{1}} \otimes \cdots \otimes \mathscr{A}^{v_{n}}$ of such spaces. It decomposes under the Lie algebra action as

$$
\mathscr{A}^{v_{1}} \otimes \cdots \otimes \mathscr{A}^{v_{n}}=\bigoplus_{s=0}^{\infty}\left(\begin{array}{c}
n+s-2 \\
n-2
\end{array}\right) \mathscr{A}^{|v|+2 s},
$$

(where $\left.|v|=\sum v_{i}\right)$, that is, $\mathscr{A}^{|v|+2 s}$ occurs with the multiplicity $\left(\begin{array}{c}n+s-2 \\ n-2\end{array}\right)$. A highest weight vector $Q$ in $\mathscr{A}^{v_{1}} \otimes \cdots \otimes \mathscr{A}^{v_{n}}$ of weight $-(|\nu|+2 s)$ is an image of 1 under an intertwining embedding

$$
\mathscr{A}^{|v|+2 s} \rightarrow \mathscr{A}^{v_{1}} \otimes \cdots \otimes \mathscr{A}^{v_{n}}
$$

In our realization this means that $Q$ is a homogeneous polynomial of degree $s$ which can be expressed as a function of the differences $z_{i}-z_{j}$ of the coordinates. Equivalently, $Q$ satisfies

$$
Q\left(a z_{1}+b, \ldots, a z_{n}+b\right)=a^{s} Q\left(z_{1}, \ldots, z_{n}\right), \quad a, b \in \mathbb{C}
$$

In agreement with (1.7), the space of such polynomials has dimension $\left(\begin{array}{c}n+s-2 \\ n-2\end{array}\right)$.

Let, for each highest weight vector $Q, \mathscr{E}_{Q}$ be the intertwining operator

$$
\Phi_{Q}: \mathscr{A}^{v_{1}} \otimes \cdots \otimes \mathscr{A}^{v_{n}} \longrightarrow \mathscr{A}^{|v|+2 s}
$$

determined by $\mathscr{E}_{Q}^{*} 1=Q$. In the present realization, $\mathscr{F}_{Q}$ is a differential operator. In fact, if

$$
Q(z)=\sum_{|t|=s} c_{t} z_{1}^{t_{1}} \cdots z_{n}^{t_{n}}
$$


then

$$
\mathscr{F}_{Q}\left(f_{1} \otimes \cdots \otimes f_{n}\right)(z)=\sum_{|t|=s} \bar{c}_{t} \frac{f_{1}^{\left(t_{1}\right)}(z) \cdots f_{n}^{\left(t_{n}\right)}(z)}{\left(v_{1}\right)_{t_{1}} \cdots\left(v_{n}\right)_{t_{n}}} .
$$

In particular, if $n=2$ and $Q\left(z_{1}, z_{2}\right)=\left(z_{1}-z_{2}\right)^{s}$ then

$$
\mathscr{F}_{Q}\left(f_{1} \otimes f_{2}\right)(z)=\sum_{k=0}^{s}\left(\begin{array}{l}
s \\
k
\end{array}\right) \frac{(-1)^{s-k}}{\left(v_{1}\right)_{k}\left(v_{2}\right)_{s-k}} f_{1}^{(k)}(z) f_{2}^{(s-k)}(z) .
$$

These bilinear operators occur (although usually expressed in homogeneous coordinates (cf. Appendix A) and with $v_{i}$ replaced by negative integers) in classical invariant theory and are called transvectants. They have also been used in the theory of modular forms, where they are called Rankin-Cohen brackets [26, 43].

It follows from (1.12) that, writing $e_{m}(z)=z^{m}$,

$$
\mathscr{F}_{Q}\left(e_{m_{1}} \otimes \cdots \otimes e_{m_{n}}\right)=\overline{T_{1} Q(m)} e_{|m|-s},
$$

where $T_{1} Q$ is the polynomial

$$
T_{1} Q\left(m_{1}, \ldots, m_{n}\right)=(-1)^{s} \sum_{|t|=s} c_{t} \frac{\left(-m_{1}\right)_{t_{1}} \cdots\left(-m_{n}\right)_{t_{n}}}{\left(v_{1}\right)_{t_{1}} \cdots\left(v_{n}\right)_{t_{n}}} .
$$

Since the monomials are eigenfunctions of the rotations of the disc, this exhibits $T_{1} Q$ as a coupling coefficient, with respect to this subgroup, of our representation. If $Q^{\prime}$ is another highest weight vector then, by an application of Schur's lemma to $\mathscr{F}_{Q^{\prime}} \mathscr{F}_{Q}^{*}$,

$$
\sum_{|m|=k+s} \frac{\left(v_{1}\right)_{m_{1}} \cdots\left(v_{n}\right)_{m_{n}}}{m_{1} ! \cdots m_{n} !} T_{1} Q(m) \overline{T_{1} Q^{\prime}(m)}=\frac{(|v|+2 s)_{k}}{k !}\left\langle Q, Q^{\prime}\right\rangle_{\otimes_{i=1}^{n} A^{v_{i}}}
$$

for $k=0,1,2, \ldots$. Thus, given an orthogonal system of highest weight vectors, the transform $T_{1}$ gives a corresponding system of discrete orthogonal polynomials. For fixed $k$ one may eliminate one variable and view these as polynomials of $n-1$ variables. In the case $n=2$ one obtains in this way the Hahn polynomials.

Replacing the monomials by formal eigenvectors to other one-parameter subgroups gives multivariable Jacobi polynomials

$$
T_{2} Q\left(x_{1}, \ldots, x_{n}\right)=\sum_{|t|=s} c_{t} \frac{x_{1}^{t_{1}} \cdots x_{n}^{t_{n}}}{\left(v_{1}\right)_{t_{1}} \cdots\left(v_{n}\right)_{t_{n}}}
$$

and multivariable continuous Hahn polynomials

$$
T_{3} Q\left(x_{1}, \ldots, x_{n}\right)=\sum_{|t|=s} c_{t} \frac{\left(v_{1} / 2-i x_{1}\right)_{t_{1}} \cdots\left(v_{n} / 2-i x_{n}\right)_{t_{n}}}{\left(v_{1}\right)_{t_{1}} \cdots\left(v_{n}\right)_{t_{n}}} .
$$

Many orthogonal and biorthogonal polynomial systems occurring in the literature may be obtained by applying the three transforms $T_{i}$ to specific bases in the space of highest weight vectors; confer [31] and the references given there.

In this paper, we will obtain a $q$-analogue of the transform $T_{1}$, leading to multivariable $q$-Hahn polynomials. One may also consider more general coupling coefficients 
connected with the so-called twisted primitive elements of the quantum algebra. This leads to multivariable generalizations of Askey-Wilson polynomials and $q$-Racah polynomials; confer [32] for some further remarks.

The plan of the paper is as follows. Section 2 contains preliminaries on $q$-series and quantum algebra. In Section 3 we find a $q$-analogue of the expression (1.12). This is used in Section 4 to find analogues of the transform $T_{1}$ and of the orthogonality relations (1.16). In Section 5 we study $q$-analogues of the coupling kernels introduced in [31]. These are the reproducing kernels for spaces of coupling coefficients. In Section 6 we discuss identities involving multivariable $q$-Hahn polynomials and coupling kernels which follow from the quantum algebraic interpretation. In Section 7 we consider explicit examples of orthogonal and biorthogonal systems which may be obtained as coupling coefficients. There are two appendices, where we use quasi-commuting homogeneous coordinates to give an algebraic description of the space of highest weight vectors.

\section{Preliminaries}

2.1. Notation. Throughout the paper, $q$ will be a fixed number in the range $0<q<1$. It will be convenient to use the symmetric $q$-factorials and $q$-Pochhammer symbols defined by

$$
[k] !=[1][2] \cdots[k], \quad[a]_{k}=[a][a+1] \cdots[a+k-1],
$$

where

$$
[a]=\frac{q^{a / 2}-q^{-a / 2}}{q^{1 / 2}-q^{-1 / 2}} .
$$

This is related to the more standard notation of [10],

$$
(a ; q)_{k}=(1-a)(1-a q) \cdots\left(1-a q^{k-1}\right),
$$

by

$$
[a]_{k}=\frac{\left(q^{a} ; q\right)_{k}}{(1-q)^{k}} q^{-(1 / 4) k(2 a+k-3)}, \quad \frac{[a]_{k}}{[b]_{k}}=\frac{\left(q^{a} ; q\right)_{k}}{\left(q^{b} ; q\right)_{k}} q^{(1 / 2) k(b-a)} .
$$

Sometimes we will use the multi-index notation

$$
[a]_{k}=\left[a_{1}\right]_{k_{1}} \cdots\left[a_{n}\right]_{k_{n}}, \quad[k] !=\left[k_{1}\right] ! \cdots\left[k_{n}\right] ! .
$$

The basic hypergeometric series ${ }_{r} \phi_{s}$ is defined by [10]

$$
{ }_{r} \phi_{s}\left[\begin{array}{l}
a_{1}, \ldots, a_{r} \\
b_{1}, \ldots, b_{s}
\end{array} ;, z\right]=\sum_{k=0}^{\infty} \frac{\left(a_{1} ; q\right)_{k} \cdots\left(a_{r} ; q\right)_{k}}{(q ; q)_{k}\left(b_{1} ; q\right)_{k} \cdots\left(b_{s} ; q\right)_{k}}\left((-1)^{k} q^{\left(\begin{array}{c}
k \\
2
\end{array}\right)}\right)^{1+s-r} z^{k}
$$

in particular,

$$
{ }_{r+1} \phi_{r}\left[\begin{array}{c}
q^{a_{1}}, \ldots, q^{a_{r+1}} \\
q^{b_{1}}, \ldots, q^{b_{r}} ; q, z
\end{array}\right]=\sum_{k=0}^{\infty} \frac{\left[a_{1}\right]_{k} \cdots\left[a_{r+1}\right]_{k}}{[k] !\left[b_{1}\right]_{k} \cdots\left[b_{r}\right]_{k}} q^{(1 / 2) k(|a|-|b|-1)} z^{k},
$$


where $|x|=\sum x_{i}$. The $q$-Hahn polynomials, introduced by Hahn [12], are given by

$$
Q_{n}\left(q^{-x} ; \alpha, \beta, N \mid q\right)={ }_{3} \phi_{2}\left[\begin{array}{c}
q^{-n}, \alpha \beta q^{n+1}, q^{-x} \\
\alpha q, q^{-N}
\end{array} ; q, q\right], \quad n=0,1, \ldots, N
$$

this is a polynomial of degree $n$ in $q^{-x}$. They form an orthogonal system with respect to a measure supported on $\left\{1, q, \ldots, q^{-N}\right\}$; confer [18]. The dual orthogonality relation may be written as an orthogonality relation for a different system of polynomials, namely, the dual $q$-Hahn polynomials.

We will write

$$
D_{q} f(z)=\frac{f\left(q^{1 / 2} z\right)-f\left(q^{-1 / 2} z\right)}{q^{1 / 2} z-q^{-1 / 2} z}, \quad f^{[k]}(z)=D_{q}^{k} f(z)
$$

for the symmetric $q$-derivative. It satisfies the $q$-Leibniz rule

$$
D_{q}(f g)(z)=\left(D_{q} f\right)(z) g\left(q^{-1 / 2} z\right)+f\left(q^{1 / 2} z\right)\left(D_{q} g\right)(z)
$$

We will need the following simple lemma in a special case.

LEMMA 2.1. For $\alpha_{i j}$ scalars, $f_{i}$ formal power series and $z_{i}, w_{i}$ formal variables, the following identity holds:

$$
\begin{aligned}
& \sum_{k_{1}, \ldots, k_{n}=0}^{\infty} \frac{w_{1}^{k_{1}} \cdots w_{n}^{k_{n}}}{\left[k_{1}\right] ! \cdots\left[k_{n}\right] !} f_{1}^{\left[k_{1}\right]}\left(\alpha_{11}^{k_{1}} \cdots \alpha_{1 n}^{k_{n}} z_{1}\right) \cdots f_{n}^{\left[k_{n}\right]}\left(\alpha_{n 1}^{k_{1}} \cdots \alpha_{n n}^{k_{n}} z_{n}\right) \\
& \quad=\sum_{k_{1}, \ldots, k_{n}=0}^{\infty} \frac{z_{1}^{k_{1}} \cdots z_{n}^{k_{n}}}{\left[k_{1}\right] ! \cdots\left[k_{n}\right] !} f_{1}^{\left[k_{1}\right]}\left(\alpha_{11}^{k_{1}} \cdots \alpha_{n 1}^{k_{n}} w_{1}\right) \cdots f_{n}^{\left[k_{n}\right]}\left(\alpha_{1 n}^{k_{1}} \cdots \alpha_{n n}^{k_{n}} w_{n}\right) .
\end{aligned}
$$

Proof. It suffices to take $f_{i}(z)=z^{m_{i}}$. Since then

$$
f_{i}^{\left[k_{i}\right]}(z)=\frac{\left[m_{i}\right] !}{\left[m_{i}-k_{i}\right] !} z^{m_{i}-k_{i}}
$$

the left-hand side is given by

$$
\sum_{k_{1}, \ldots, k_{n}=0}^{m_{1}, \ldots, m_{n}} \frac{\left[m_{1}\right] ! \cdots\left[m_{n}\right] !}{\left[k_{1}\right] !\left[m_{1}-k_{1}\right] ! \cdots\left[k_{n}\right] !\left[m_{n}-k_{n}\right] !} w_{1}^{k_{1}} \cdots w_{n}^{k_{n}} z_{1}^{m_{1}-k_{1}} \cdots z_{n}^{m_{n}-k_{n}} \prod_{i, j=1}^{n} \alpha_{i j}^{k_{j}\left(m_{i}-k_{i}\right)}
$$

Changing $k$ to $m-k$ gives the same expression with $z$ and $w$ interchanged and $\alpha_{i j}$ replaced by $\alpha_{j i}$. This proves the lemma.

For any $x \in \mathbb{R}^{n}$, we will denote by $x^{*}$ the element in $\mathbb{R}^{n}$ with coordinates

$$
x_{i}^{*}=\sum_{j=1}^{i-1} x_{j}-\sum_{j=i+1}^{n} x_{j}, \quad i=1, \ldots, n .
$$


Then

$$
\{x, y\}=\sum_{i=1}^{n} x_{i} y_{i}^{*}=\sum_{i>j} x_{i} y_{j}-\sum_{i<j} x_{i} y_{j}
$$

defines a skew-symmetric form on $\mathbb{R}^{n}$. It appears naturally in connection with multivariable $q$-series; for instance, one has the multivariable $q$-Chu-Vandermonde formula (equivalent to [10, Exercise 1.3])

$$
\frac{\left[x_{1}+\cdots+x_{n}\right]_{k}}{[k] !}=\sum_{i_{1}+\cdots+i_{n}=k} \frac{\left[x_{1}\right]_{i_{1}} \cdots\left[x_{n}\right]_{i_{n}}}{\left[i_{1}\right] ! \cdots\left[i_{n}\right] !} q^{(1 / 2)\{i, x\}} .
$$

2.2. Quantum algebra. The quantum algebra (or quantized universal enveloping algebra) $U=U_{q^{1 / 2}(\mathfrak{s u}(1,1))}$ is the associative involutive algebra over the complex numbers defined by generators

$$
X_{+}, \quad X_{-}, \quad K, \quad K^{-1},
$$

relations

$$
\begin{gathered}
K K^{-1}=K^{-1} K=1, \\
K X_{ \pm} K^{-1}=q^{ \pm 1 / 2} X_{ \pm}, \\
X_{+} X_{-}-X_{-} X_{+}=\frac{K^{2}-K^{-2}}{q^{1 / 2}-q^{-1 / 2}},
\end{gathered}
$$

and involution

$$
\left(K^{ \pm}\right)^{*}=K^{ \pm}, \quad X_{ \pm}^{*}=-X_{\mp} ;
$$

here and below we write $K^{+}=K, K^{-}=K^{-1}$ when convenient. We refer to [4] for an introduction to quantum group theory. A unitary representation of $u$ is a representation by densely defined operators on a Hilbert space, such that the involution $*$ coincides with the Hilbert space adjoint.

The algebra $U$ has the additional structure of a Hopf algebra. We need to discuss only the coproduct, which is the map $\Delta: \mathcal{U} \rightarrow \mathcal{U} \otimes$ given on the generators by

$$
\Delta\left(K^{ \pm}\right)=K^{ \pm} \otimes K^{ \pm}, \quad \Delta\left(X_{ \pm}\right)=K \otimes X_{ \pm}+X_{ \pm} \otimes K^{-1} .
$$

This means that, given two unitary representations $H_{1}$ and $H_{2}$ of $u$, another one is defined on the Hilbert tensor product $H_{1} \otimes H_{2}$ by

$$
K^{ \pm}=K^{ \pm} \otimes K^{ \pm}, \quad X_{ \pm}=X_{ \pm} \otimes K+K^{-1} \otimes X_{ \pm} .
$$

The coproduct is non-cocommutative, that is, the flip $f \otimes g \mapsto g \otimes f$ is not an intertwining map $H_{1} \otimes H_{2} \rightarrow H_{2} \otimes H_{1}$. It is, however, coassociative in the sense that there is only one way to repeat it to obtain a representation on a finite tensor product $H_{1} \otimes \cdots \otimes H_{n}$, namely, by

$$
\begin{aligned}
K^{ \pm}= & K^{ \pm} \otimes \cdots \otimes K^{ \pm} \\
X_{ \pm}= & X_{ \pm} \otimes K \otimes \cdots \otimes K+K^{-1} \otimes X_{ \pm} \otimes K \otimes \cdots \otimes K \\
& +\cdots+K^{-1} \otimes \cdots \otimes K^{-1} \otimes X_{ \pm} .
\end{aligned}
$$


It is easily proved that

$$
\begin{aligned}
X_{ \pm}^{k} & =\sum_{j_{1}+\cdots+j_{n}=k} \frac{[k] !}{\left[j_{1}\right] ! \cdots\left[j_{n}\right] !} X_{ \pm}^{j_{1}} K^{j_{1}^{*}} \otimes \cdots \otimes X_{ \pm}^{j_{n}} K^{j_{n}^{*}} \\
& =\sum_{j_{1}+\cdots+j_{n}=k} \frac{[k] !}{\left[j_{1}\right] ! \cdots\left[j_{n}\right] !} K^{j_{1}^{*}} X_{ \pm}^{j_{1}} \otimes \cdots \otimes K^{j_{n}^{*}} X_{ \pm}^{j_{n}}
\end{aligned}
$$

where $*$ is given by (2.14).

2.3. Holomorphic realization. We will work with the holomorphic realization of

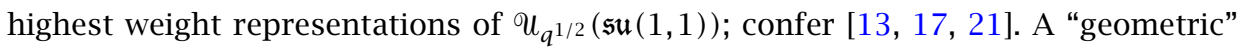
motivation for this realization is provided in Appendix A.

For $v>0$ and $0<q<1$, we denote by $\mathscr{A}_{q}^{v}$ the Hilbert space of analytic functions with the norm

$$
\|f\|^{2}=\langle f, f\rangle=\sum_{k=0}^{\infty} \frac{[k] !}{[v]_{k}}|\hat{f}(k)|^{2},
$$

where $f(z)=\sum \hat{f}(k) z^{k}$. By Cauchy's $q$-binomial formula [10], $\mathscr{A}_{q}^{v}$ has the reproducing kernel

$$
k_{w}(z)=\sum_{k=0}^{\infty} \frac{[v]_{k}}{[k] !}(z \bar{w})^{k}=\prod_{j=0}^{\infty} \frac{1-q^{1 / 2+v / 2+j} z \bar{w}}{1-q^{1 / 2-v / 2+j} z \bar{w}}
$$

(here we use that $0<q<1$ ) and thus the natural domain of definition is the disc

$$
\left\{z \in \mathbb{C} ;|z|<q^{v / 4-1 / 4}\right\} .
$$

We write $e_{k}(z)=z^{k}$ for monomials, so that

$$
\langle f, g\rangle=\sum_{k=0}^{\infty} \frac{[v]_{k}}{[k] !}\left\langle f, e_{k}\right\rangle\left\langle e_{k}, g\right\rangle .
$$

There is a unitary representation of $\bigcup_{q}(\mathfrak{s u}(1,1))$ on $\mathscr{A}_{q}^{v}$, given by

$$
\begin{aligned}
& X_{+} f(z)=-\frac{f\left(q^{1 / 2} z\right)-f\left(q^{-1 / 2} z\right)}{q^{1 / 2} z-q^{-1 / 2} z}, \\
& X_{-} f(z)=z \frac{q^{v / 2} f\left(q^{1 / 2} z\right)-q^{-v / 2} f\left(q^{-1 / 2} z\right)}{q^{1 / 2}-q^{-1 / 2}}, \\
& K^{ \pm} f(z)=q^{\mp v / 4} f\left(q^{\mp 1 / 2} z\right) .
\end{aligned}
$$

In the limit $q \rightarrow 1$,

$$
\begin{gathered}
X_{+} \longrightarrow-\frac{d}{d z}, \\
X_{-} \longrightarrow z^{2} \frac{d}{d z}+v z, \\
4 \log _{q} K^{ \pm} \longrightarrow \mp\left(v+2 z \frac{d}{d z}\right),
\end{gathered}
$$

so we recover the Lie algebra operators (1.4). 
Though we will not use it, we remark that for $v>1$ there is an integral formula for the norm of $\mathscr{A}_{q}^{v}$ [17]. In terms of Jackson's $q$-integral [10]

$$
\int_{0}^{c} f(x) d_{q} x=c(1-q) \sum_{k=0}^{\infty} q^{k} f\left(q^{k} c\right)
$$

it can be written as

$$
\|f\|^{2}=\frac{[v-1]\left(1+q^{-1 / 2}\right)}{2 \pi} \int_{-\pi}^{\pi} \int_{0}^{q^{(1 / 4)(v-1)}} r\left|f\left(r e^{i \theta}\right)\right|^{2} \prod_{j=0}^{\infty} \frac{1-r^{2} q^{(1 / 2)(3-v)+j}}{1-r^{2} q^{(1 / 2)(v-1)+j}} d_{q^{1 / 2}} r d \theta
$$

which is a $q$-analogue of (1.2). In contrast to the case $q=1$, this formula extends to the case $0<v<1$, though the mass on the outer circle $|z|=q^{(1 / 4)(v-1)}$ is then negative.

3. Quantum transvectants. In this section we will obtain an expression for the multilinear transvectants in the present context. As in the case $q=1$, there is a decomposition

$$
A_{q}^{v_{1}} \otimes \cdots \otimes \mathscr{A}_{q}^{v_{n}}=\bigoplus_{s=0}^{\infty}\left(\begin{array}{c}
n+s-2 \\
n-2
\end{array}\right) \mathscr{A}_{q}^{|v|+2 s},
$$

where $|v|=\sum v_{i}$. We define a transvectant of order $s$ to be an intertwining map

$$
\mathscr{F}: \mathscr{A}_{q}^{v_{1}} \otimes \cdots \otimes \mathscr{A}_{q}^{v_{n}} \longrightarrow \mathscr{A}_{q}^{|v|+2 s}
$$

thus the transvectants of order $s$ form a linear space of dimension $\left(\begin{array}{c}n+s-2 \\ n-2\end{array}\right)$.

A highest weight vector is, by definition, a solution in $\bigotimes_{i=1}^{n} A_{q}^{v_{i}}$ to

$$
K Q=q^{(-1 / 4)(|v|+2 s)} Q, \quad X_{+} Q=0 .
$$

These are mapped to constants by the transvectants of order $s$. The first equation means that they are homogeneous polynomials of degree $s$. For each highest weight vector $Q$, we denote by $\mathscr{F}_{Q}$ the transvectant of order $s$ determined by $\mathscr{F}_{Q}^{*} 1=Q$. This gives a one-to-one correspondence between highest weight vectors and transvectants.

We will now fix $Q$ and seek a $q$-analogue of the expression (1.12) for $\mathscr{F}_{Q}$. It will be convenient to write $\mu=|\nu|+2 s$,

$$
\langle f, g\rangle_{v}=\langle f, g\rangle_{A_{q}^{\nu_{1}} \otimes \cdots \otimes \mathcal{H}_{q}^{\nu_{n}}}, \quad\langle f, g\rangle_{\mu}=\langle f, g\rangle_{\mathscr{A}_{q}^{\mu}} .
$$

Since

$$
e_{k}=\frac{1}{[\mu]_{k}} X_{-}^{k} 1
$$

in $\mathscr{A}_{q}^{\mu}$, we must have

$$
\mathscr{F}_{Q}^{*} e_{k}=\frac{1}{[\mu]_{k}} X_{-}^{k} Q .
$$

For $f$ a polynomial (to avoid questions of convergence) in $\bigotimes_{i=1}^{n} A_{q}^{v_{i}}$, it follows that

$$
\begin{aligned}
\mathscr{g}_{Q} f(w) & =\left\langle\mathscr{E}_{Q} f, k_{w}\right\rangle_{\mu}=\sum_{k=0}^{\infty} \frac{[\mu]_{k}}{[k] !} w^{k}\left\langle\mathscr{f}_{Q} f, e_{k}\right\rangle_{\mu}=\sum_{k=0}^{\infty} \frac{[\mu]_{k}}{[k] !} w^{k}\left\langle f, \mathscr{E}_{Q}^{*} e_{k}\right\rangle_{v} \\
& =\sum_{k=0}^{\infty} \frac{w^{k}}{[k] !}\left\langle f, X_{-}^{k} Q\right\rangle_{v}=\sum_{k=0}^{\infty} \frac{(-1)^{k} w^{k}}{[k] !}\left\langle X_{+}^{k} f, Q\right\rangle_{v}=\left\langle\tau_{w} f, Q\right\rangle_{v},
\end{aligned}
$$


where $\tau_{w}$ is the $q$-translation operator

$$
\tau_{w}=\sum_{k=0}^{\infty} \frac{\left(-w X_{+}\right)^{k}}{[k] !} .
$$

LEMMA 3.1. As an operator on the subspace of polynomials in $\bigotimes_{i=1}^{n} A_{q}^{v_{i}}, \tau_{w}$ is given by

$$
\begin{aligned}
\boldsymbol{T}_{w} & \left(f_{1} \otimes \cdots \otimes f_{n}\right)(z) \\
& =\sum_{k_{1}, \ldots, k_{n}=0}^{\infty} \frac{w^{k_{1}+\cdots+k_{n}}}{\left[k_{1}\right] ! \cdots\left[k_{n}\right] !} q^{(1 / 4)\{k, v\}} f_{1}^{\left[k_{1}\right]}\left(q^{-(1 / 2) k_{1}^{*}} z_{1}\right) \cdots f_{n}^{\left[k_{n}\right]}\left(q^{-(1 / 2) k_{n}^{*}} z_{n}\right) \\
& =\sum_{k_{1}, \ldots, k_{n}=0}^{\infty} \frac{z_{1}^{k_{1}} \cdots z_{n}^{k_{n}}}{\left[k_{1}\right] ! \cdots\left[k_{n}\right] !} f_{1}^{\left[k_{1}\right]}\left(q^{(1 / 2) k_{1}^{*}+(1 / 4) v_{1}^{*}} w\right) \cdots f_{n}^{\left[k_{n}\right]}\left(q^{(1 / 2) k_{n}^{*}+(1 / 4) v_{n}^{*}} w\right) .
\end{aligned}
$$

Proof. The first expression follows from (2.23). The second one then follows from Lemma 2.1, in the case when

$$
\alpha_{i j}= \begin{cases}q, & i<j, \\ 1, & i=j, \\ q^{-1}, & i>j\end{cases}
$$

Plugging the last expression of the lemma into the equality

$$
\mathscr{S}_{Q}\left(f_{1} \otimes \cdots \otimes f_{n}\right)(w)=\left\langle\tau_{w}\left(f_{1} \otimes \cdots \otimes f_{n}\right), Q\right\rangle_{v}
$$

gives the following explicit expression for the transvectant.

THEOREM 3.2. If

$$
Q\left(z_{1}, \ldots, z_{n}\right)=\sum_{|t|=s} c_{t} z_{1}^{t_{1}} \cdots z_{n}^{t_{n}}
$$

is a highest weight vector, then

$$
\begin{aligned}
& \mathscr{g}_{Q}\left(f_{1} \otimes \cdots \otimes f_{n}\right)(w) \\
& \quad=\sum_{|t|=s} \frac{\bar{c}_{t}}{\left[v_{1}\right]_{t_{1}} \cdots\left[v_{n}\right]_{t_{n}}} f_{1}^{\left[t_{1}\right]}\left(q^{(1 / 4) v_{1}^{*}+(1 / 2) t_{1}^{*}} w\right) \cdots f_{n}^{\left[t_{n}\right]}\left(q^{(1 / 4) v_{n}^{*}+(1 / 2) t_{n}^{*}} w\right),
\end{aligned}
$$

where $*$ is defined by (2.14).

As an example, for $n=2$ the space of highest weight vectors of degree $s$ is onedimensional. It is easy to verify that it is spanned by

$$
\begin{aligned}
Q\left(z_{1}, z_{2}\right) & =\prod_{j=0}^{s-1}\left(q^{\nu_{2} / 4+j / 2} z_{1}-q^{-v_{1} / 4-j / 2} z_{2}\right) \\
& =\sum_{i+j=s} \frac{[s] !}{[i] ![j] !}(-1)^{j} q^{(1 / 4)\left((s-1)(i-j)+v_{2} i-v_{1} j\right)} z_{1}^{i} z_{2}^{j}
\end{aligned}
$$


confer also Section 7 and Appendix B. The corresponding transvectant is

$$
\begin{aligned}
& \mathscr{E}_{Q}\left(f_{1} \otimes f_{2}\right)(w) \\
& \quad=\sum_{i+j=s} \frac{[s] !}{[i] ![j] !}(-1)^{j} q^{(1 / 4)\left((s-1)(i-j)+v_{2} i-v_{1} j\right)} \frac{f_{1}^{[i]}\left(q^{-v_{2} / 4-j / 2} w\right) f_{2}^{[j]}\left(q^{v_{1} / 4+i / 2} w\right)}{\left[v_{1}\right]_{i}\left[v_{2}\right]_{j}},
\end{aligned}
$$

which is a $q$-analogue of (1.13).

4. Coupling coefficients. To each highest weight vector $Q$ we associate the function $P$ on $\mathbb{Z}_{+}^{n}$ given by

$$
\mathscr{I}_{Q}\left(e_{m_{1}} \otimes \cdots \otimes e_{m_{n}}\right)=\overline{P\left(m_{1}, \ldots, m_{n}\right)} e_{|m|-s},
$$

where $s$ is the degree of $Q$. Equivalently,

$$
\frac{[|v|+2 s]_{k}}{[k] !} \mathscr{F}_{Q}^{*} e_{k}=\sum_{|m|=k+s} \frac{\left[v_{1}\right]_{m_{1}} \cdots\left[v_{n}\right]_{m_{n}}}{\left[m_{1}\right] ! \cdots\left[m_{n}\right] !} P(m) e_{m_{1}} \otimes \cdots \otimes e_{m_{n}} .
$$

This exhibits $P$ as a coupling coefficient for the quantum algebra. If $Q$ is given by (3.12), it follows from Theorem 3.2 that

$$
P(m)=\sum_{|t|=s} c_{t} \frac{\left[-m_{1}\right]_{t_{1}} \cdots\left[-m_{n}\right]_{t_{n}}}{\left[v_{1}\right]_{t_{1}} \cdots\left[v_{n}\right]_{t_{n}}}(-1)^{s} q^{(1 / 4)\left(v_{1}^{*}+2 t_{1}^{*}\right)\left(m_{1}-t_{1}\right)+\cdots+(1 / 4)\left(v_{n}^{*}+2 t_{n}^{*}\right)\left(m_{n}-t_{n}\right)} .
$$

Using that $\sum_{i} x_{i} x_{i}^{*}=0$, one may rewrite the exponent of $q$ as

$$
\frac{1}{8}\{v+2 m, v+2 t\}
$$

where $\{$,$\} is given by (2.15). Thus P=T Q$, where $T$ is the linear operator defined by

$$
T\left(e_{t_{1}} \otimes \cdots \otimes e_{t_{n}}\right)(m)=(-1)^{|t|} \frac{\left[-m_{1}\right]_{t_{1}} \cdots\left[-m_{n}\right]_{t_{n}}}{\left[v_{1}\right]_{t_{1}} \cdots\left[v_{n}\right]_{t_{n}}} q^{(1 / 8)\{v+2 m, v+2 t\}} .
$$

Now let $Q$ and $Q^{\prime}$ be two highest weight vectors. Then, by an application of Schur's lemma to $\mathscr{L}_{Q^{\prime}} \mathscr{F}_{Q^{*}}$,

$$
\begin{aligned}
\left\langle Q, Q^{\prime}\right\rangle\left\|e_{k}\right\|^{2} & =\left\langle\mathscr{f}_{Q}^{*} e_{k}, \mathscr{f}_{Q^{\prime}}^{*} e_{k}\right\rangle \\
& =\sum_{m_{1}, \ldots, m_{n}=0}^{\infty} \frac{\left[v_{1}\right]_{m_{1}} \cdots\left[v_{n}\right]_{m_{n}}}{\left[m_{1}\right] ! \cdots\left[m_{n}\right] !}\left\langle\mathscr{E}_{Q^{*}}^{*} e_{k}, e_{m}\right\rangle\left\langle e_{m}, \mathscr{f}_{Q^{\prime}}^{*} e_{k}\right\rangle \\
& =\sum_{|m|=k+s} \frac{\left[v_{1}\right]_{m_{1}} \cdots\left[v_{n}\right]_{m_{n}}}{\left[m_{1}\right] ! \cdots\left[m_{n}\right] !} T Q(m) \overline{T Q^{\prime}(m)}\left\|e_{k}\right\|^{4} .
\end{aligned}
$$

This gives the following orthogonality property. 
THEOREM 4.1. For $Q$ and $Q^{\prime}$ two highest weight vectors in $\bigotimes_{i=1}^{n} A_{q}^{v_{i}}$ and $k$ a nonnegative integer,

$$
\sum_{|m|=k+s} \frac{\left[v_{1}\right]_{m_{1}} \cdots\left[v_{n}\right]_{m_{n}}}{\left[m_{1}\right] ! \cdots\left[m_{n}\right] !} T Q(m) \overline{T Q^{\prime}(m)}=\frac{[|v|+2 s]_{k}}{[k] !}\left\langle Q, Q^{\prime}\right\rangle,
$$

where $s$ is the degree of $Q$ (or of $Q^{\prime}$ ).

The function $T Q$ is not a polynomial. To obtain orthogonal polynomials from Theorem 4.1, we introduce the new variables $x_{i}=q^{-\left(m_{1}+m_{2}+\cdots+m_{i}\right)}, i=1, \ldots, n$. Switching to standard notation (2.3) and using

$$
|m||t|=\{m, t\}+\sum_{i} m_{i} t_{i}+2 \sum_{i<j} m_{i} t_{j}
$$

we obtain

$$
\begin{aligned}
& q^{(-1 / 2) s|m|-(1 / 4)\{m, v\}} \operatorname{TQ}\left(m_{1}, \ldots, m_{n}\right) \\
& \quad=\sum_{|t|=s} c_{t}(-1)^{s} q^{(1 / 4)\{v, t\}+(1 / 2) \sum_{i=1}^{n} v_{i} t_{i}} x_{1}^{t_{2}} \cdots x_{n-1}^{t_{n}} \frac{\left(x_{1} ; q\right)_{t_{1}}\left(x_{2} / x_{1} ; q\right)_{t_{2}} \cdots\left(x_{n} / x_{n-1} ; q\right)_{t_{n}}}{\left(q^{v_{1}} ; q\right)_{t_{1}}\left(q^{v_{2}} ; q\right)_{t_{2}} \cdots\left(q^{v_{n}} ; q\right)_{t_{n}}},
\end{aligned}
$$

which is a polynomial in the variables $x_{i}$ of total degree $|t|$. Given a complete orthogonal (biorthogonal) family of highest weight vectors, Theorem 4.1 gives an orthogonality (biorthogonality) relation for the corresponding polynomials. For fixed $k$, one may view this as a system of polynomials in the $n-1$ variables $x_{1}, \ldots, x_{n-1}$. By a dimension count, the latter system will be complete.

In particular, for $n=2$ and $Q$ as in (3.14) one has

$$
\begin{aligned}
T Q\left(m_{1}, m_{2}\right)= & q^{(1 / 4)\left(v_{1} m_{2}-v_{2} m_{1}\right)} \\
& \times \sum_{i+j=s} \frac{[s] !\left[-m_{1}\right]_{i}\left[-m_{2}\right]_{j}}{[i] ![j] !\left[v_{1}\right]_{i}\left[v_{2}\right]_{j}}(-1)^{i} q^{(1 / 4)(s-1)(i-j)+(1 / 2) i\left(v_{2}+m_{2}\right)-(1 / 2) j\left(v_{1}+m_{1}\right)} .
\end{aligned}
$$

Using transformation formulas from [10] one may check that

$$
\begin{aligned}
& q^{-(1 / 2) s\left(m_{1}+m_{2}\right)-(1 / 4)\left(v_{1} m_{2}-v_{2} m_{1}\right)} T Q\left(m_{1}, m_{2}\right) \\
& \quad=q^{(1 / 2) s\left(v_{2}-v_{1}\right)-(1 / 4) s(s-1)} \frac{\left(q^{-m_{1}-m_{2}} ; q\right)_{s}}{\left(q^{v_{2}} ; q\right)_{s}} Q_{s}\left(q^{-m_{1}} ; q^{v_{1}-1}, q^{v_{2}-1}, m_{1}+m_{2} \mid q\right),
\end{aligned}
$$

where the right-hand side is a $q$-Hahn polynomial as defined in (2.8). Theorem 4.1 then gives the orthogonality relation for $q$-Hahn polynomials; confer [22, 34].

5. Coupling kernels. In [31] we introduced certain functions called coupling kernels. We expressed them explicitly as multivariable hypergeometric sums. The discrete coupling kernels, connected with the transform $T_{1}$, were used in [30] to study Wigner $9 j$-symbols. The coupling kernels connected with $T_{2}$ were independently introduced 
by Xu [42], who used them to study Cesàro summability of multivariable Jacobi polynomial expansions. In this section we will generalize the explicit expression for discrete coupling kernels to the quantum algebra case.

Just as coupling coefficients are matrix elements for the intertwining maps $\oiint_{Q}$ : $\bigotimes_{i=1}^{n} A_{q}^{v_{i}} \rightarrow A_{q}^{|v|+2 s}$, coupling kernels are matrix elements for the intertwining projection $\Pi_{s}$ from $\bigotimes_{i=1}^{n} A_{q}^{v_{i}}$ onto the isotypic subspace equivalent to $\left(\begin{array}{c}n+s-2 \\ n-2\end{array}\right) \mathscr{A}_{q}^{|v|+2 s}$. More precisely, for any elements $X, Y$ of the quantum algebra, consider the matrix element

$$
\left\langle\Pi_{s} X e_{k}, Y e_{l}\right\rangle=\left\langle\Pi_{s} X\left(e_{k_{1}} \otimes \cdots \otimes e_{k_{n}}\right), Y\left(e_{l_{1}} \otimes \cdots \otimes e_{l_{n}}\right)\right\rangle_{\otimes_{i=1}^{n} A_{q}^{v_{i}}}
$$

of $\Pi_{s}$. Let $\left(Q_{j}\right)_{j}$ be an orthonormal basis of the space $\mathscr{V}_{s}$ of highest weight vectors of degree $s$. Then, by Hilbert space arguments,

$$
\Pi_{s}=\sum_{j} \mathscr{F}_{Q_{j}}^{*} \mathscr{F}_{Q_{j}}
$$

and thus

$$
\left\langle\Pi_{s} X e_{k}, Y e_{l}\right\rangle=P_{s}(k, l)\left\langle X e_{|k|-s}, Y e_{|l|-s}\right\rangle_{s l_{q}^{|v|+2 s}},
$$

where

$$
P_{S}(k, l)=\sum_{j} T Q_{j}(k) \overline{T Q_{j}(l)}
$$

We call the functions $P_{s}$ coupling kernels. Since

$$
Q_{s}(z, \bar{w})=\sum_{j} Q_{j}(z) \overline{Q_{j}(w)}
$$

is the reproducing kernel for the space $\mathscr{V}_{s}$, it follows from Theorem 4.1 that $P_{s}$ is the reproducing kernel for the corresponding space of coupling coefficients, with the reproducing property

$$
\frac{[|v|+2 s]_{k}}{[k] !} T Q(l)=\sum_{|m|=k+s} \frac{\left[v_{1}\right]_{m_{1}} \cdots\left[v_{n}\right]_{m_{n}}}{\left[m_{1}\right] ! \cdots\left[m_{n}\right] !} T Q(m) P_{s}(l, m)
$$

for $k=0,1,2, \ldots$ and $Q \in \mathscr{V}_{s}$. Choosing $Q=\sum_{j} \overline{T Q_{j}\left(l^{\prime}\right)} Q_{j}$, so that $T Q(l)=P_{s}\left(l, l^{\prime}\right)$, gives the addition formula

$$
\frac{[|v|+2 s]_{k}}{[k] !} P_{s}\left(l, l^{\prime}\right)=\sum_{|m|=k+s} \frac{\left[v_{1}\right]_{m_{1}} \cdots\left[v_{n}\right]_{m_{n}}}{\left[m_{1}\right] ! \cdots\left[m_{n}\right] !} P_{s}(l, m) P_{s}\left(m, l^{\prime}\right)
$$

for coupling kernels. Since, again by Hilbert space arguments,

$$
Q_{s}(z, \bar{w})=\sum_{|t|=|u|=s} \frac{[v]_{t}[v]_{u}}{[t] ![u] !}\left\langle\Pi_{s} e_{t}, e_{u}\right\rangle z^{t} \bar{w}^{u}
$$


we may also write

$$
P_{s}(l, m)=\sum_{|t|=|u|=s} \frac{[-l]_{t}[-m]_{u}}{[t] ![u] !}\left\langle\Pi_{s} e_{t}, e_{u}\right\rangle q^{(1 / 8)\{v+2 l, v+2 t\}+(1 / 8)\{v+2 m, v+2 u\}} .
$$

We will use this identity to prove the following theorem.

THEOREM 5.1. The coupling kernel $P_{s}$ is given by

$$
\begin{aligned}
P_{s}(l, m)= & \frac{(-1)^{s}[-|l|]_{s}[-|m|]_{s}}{[|v|+s-1]_{s}[s] !} \\
& \times \sum_{j_{1}, \ldots, j_{n}=0}^{\infty} \frac{[|v|+s-1]_{|j|}[-s]_{|j|}}{[-|l|]_{|j|}[-|m|]_{|j|}} \frac{[-l]_{j}[-m]_{j}}{[v]_{j}[j] !} q^{(1 / 4)\{v+l+m, v+2 j\}},
\end{aligned}
$$

with the convention $0 / 0=0$, so that the sum is actually finite.

We remark that for $n=2$ the space of coupling coefficients is one-dimensional, and therefore its reproducing kernel $P_{s}$ factors as a product of two $q$-Hahn polynomials. This gives a quantum algebraic proof of Rahman's Watson-type product formula for the $q$-Hahn polynomials [28].

The proof of Theorem 5.1 is similar to the case $q=1$ treated in [31]. It is based on the following projection formula.

LEMMA 5.2. Let $\mathscr{H}_{s}$ denote the subspace of $\bigotimes_{i=1}^{n} \mathscr{A}_{q}^{v_{i}}$ consisting of homogeneous polynomials of degree $s$. Then the restriction of $\Pi_{s}$ to $\mathcal{H}_{s}$ is given by

$$
\sum_{k=0}^{s} \frac{(-1)^{k}}{[k] ![2-|v|-2 s]_{k}} X_{-}^{k} X_{+}^{k}
$$

Since we failed to do so in [31], we give some history of this type of formulas. In the case $q=1$ and the realization of $\mathfrak{s u}(1,1)$ coming from spherical harmonics $\left(X_{+}=\Delta\right.$, $X_{-}=\sum_{i} x_{i}^{2}$ generate a realization of $\left(\mathscr{A}^{1 / 2} \oplus \mathscr{A}^{3 / 2}\right)^{\otimes n}$ on $\left.\mathrm{L}^{2}\left(\mathbb{R}^{n}\right)\right)$, Lemma 5.2 goes back to Clebsch [5]; confer also [7]. For su(2), it was rediscovered and used by Löwdin [23] in the context of quantum physics. This was the starting point for projection operator methods in quantum physics and representation theory, developed by Ašerova, Smirnov and Tolstoy; confer [2, 35, 37].

We indicate the simple proof of Lemma 5.2. Using the fact that $\mathscr{H}_{s}$ is an eigenspace of $K^{ \pm}$and the elementary identity

$$
[a]+[b][a+b+1]=[b+1][a+b],
$$

one proves by induction on $k$ that

$$
\left.X_{+} X_{-}^{k}\right|_{\mathscr{H}_{s}}=X_{-}^{k} X_{+}-[k][|v|+2 s+k-1] X_{-}^{k-1} .
$$

We note that the restriction of $\Pi_{s}$ to $\mathscr{H}_{s}$ is the orthogonal projection onto the subspace $\mathscr{V}_{s}$ of highest weight vectors. Denoting the operator in (5.11) by $A$, it follows from (5.13) that $X_{+} A=0$, so that $A \mathscr{H}_{s} \subseteq \mathscr{V}_{s}$. On the other hand, the image of Id $-A$ is in $X_{-} \mathscr{H}_{s-1}$, 
which is the orthogonal complement of $\mathscr{V}_{s}$ in $\mathscr{H}_{s}$. Thus $A$ is indeed the orthogonal projection onto $\mathscr{V}_{s}$.

Now let $t$ and $u$ be two multi-indices of length $s$. We want to compute the scalar product $\left\langle\Pi_{s} e_{t}, e_{u}\right\rangle$ occurring in (5.9). Since $X_{-}^{*}=-X_{+}$, Lemma 5.2 gives

$$
\left\langle\Pi_{s} e_{t}, e_{u}\right\rangle=\sum_{k=0}^{s} \frac{1}{[k] ![2-|v|-2 s]_{k}}\left\langle X_{+}^{k} e_{t}, X_{+}^{k} e_{u}\right\rangle
$$

Now, by (2.23),

$$
\begin{aligned}
X_{+}^{k} e_{t} & =\sum_{|j|=k} \frac{[k] !}{[j] !}[-t]_{j} q^{(1 / 4)\{j, v+2 t\}} e_{t-j} \\
& =(-1)^{|t|} \sum_{|j|=|t|-k} \frac{[k] !}{[j] !}[-t]_{j} q^{(1 / 4)\{t-j, v+2 t\}} e_{j},
\end{aligned}
$$

which gives

$$
\left\langle\Pi_{s} e_{t}, e_{u}\right\rangle=\sum_{j} \frac{[s-|j|] !}{[2-|v|-2 s]_{s-|j|}} \frac{[-t]_{j}[-u]_{j}}{[j] ![v]_{j}} q^{(1 / 4)\{t-j, v+2 t\}+(1 / 4)\{u-j, v+2 u\}}
$$

Inserting this expression in (5.9) gives

$$
\begin{aligned}
P_{s}(l, m)= & \sum_{|t|=|u|=s} \sum_{j} \frac{[s-|j|] !}{[2-|v|-2 s]_{s-|j|}} \frac{[-l]_{t}[-m]_{u}}{[t] ![u] !} \frac{[-t]_{j}[-u]_{j}}{[j] ![v]_{j}} \\
& \times q^{(1 / 4)\{t-j, v+2 t\}+(1 / 4)\{u-j, v+2 u\}+(1 / 8)\{v+2 l, v+2 t\}+(1 / 8)\{v+2 m, v+2 u\}} .
\end{aligned}
$$

Replace $t, u$ by $t+j, u+j$. The exponent of $q$ may then be expressed as

$$
\frac{1}{4}\{v+l+m, v+2 j\}+\frac{1}{2}\{t, j-l\}+\frac{1}{2}\{u, j-m\} .
$$

Thus, changing the order of summation, we obtain

$$
\begin{aligned}
P_{S}(l, m)= & \sum_{j} \frac{[s-|j|] !}{[2-|v|-2 s]_{s-|j|}} \frac{[-l]_{j}[-m]_{j}}{[j] ![v]_{j}} q^{(1 / 4)\{v+l+m, v+2 j\}} \\
& \times \sum_{|t|=s-|j|} \frac{[j-l]_{t}}{[t] !} q^{(1 / 2)\{t, j-l\}} \sum_{|u|=s-|j|} \frac{[j-m]_{u}}{[u] !} q^{(1 / 2)\{u, j-m\}},
\end{aligned}
$$

where the inner sums are of the form (2.16). This leads to the expression

$$
P_{s}(l, m)=\sum_{j} \frac{[|j|-|l|]_{s-|j|}[|j|-|m|]_{s-|j|}}{[2-|v|-2 s]_{s-|j|}[s-|j|] !} \frac{[-l]_{j}[-m]_{j}}{[j] ![v]_{j}} q^{(1 / 4)\{v+l+m, v+2 j\}},
$$

which may be rewritten as in Theorem 5.1. 
6. Convolution and linearization formulas. In the case $q=1$, matrix elements for the group action in the basis of monomials are given by Meixner polynomials [18]

$$
M_{n}(k ; v, c)={ }_{2} F_{1}\left(\begin{array}{c}
-n,-k \\
v
\end{array} \mid 1-\frac{1}{c}\right),
$$

where ${ }_{2} F_{1}$ is Gauss' hypergeometric function. The interpretation of the polynomials $T_{1} Q$ as coupling coefficients leads to the convolution formula

$$
\begin{aligned}
& \frac{(|v|+2 s)_{k}}{k !} T_{1} Q(l) c^{s}{ }_{2} F_{1}\left(\begin{array}{c}
-k, s-|l| \\
|v|+2 s
\end{array} \mid c\right) \\
& \quad=\sum_{|m|=k+s} \frac{\left(v_{1}\right)_{m_{1}} \cdots\left(v_{n}\right)_{m_{n}}}{m_{1} ! \cdots m_{n} !} T_{1} Q(m) \prod_{j=1}^{n}{ }_{2} F_{1}\left(\begin{array}{c}
-m_{j},-l_{j} \\
v_{j}
\end{array} \mid c\right)
\end{aligned}
$$

and the linearization formula

$$
\prod_{j=1}^{n}{ }_{2} F_{1}\left(\begin{array}{c}
-k_{j},-l_{j} \\
v_{j}
\end{array} \mid c\right)=\sum_{s=0}^{\min (|k|,|l|)} P_{s}(k, l) c^{s}{ }_{2} F_{1}\left(\begin{array}{c}
s-|k|, s-|l| \\
|v|+2 s
\end{array} \mid c\right)
$$

for Meixner polynomials, confer [31]. In this section we will generalize these formulas to the present setting.

Let $\tau_{\lambda}$ be the $q$-translation operator occurring in Lemma 3.1. We will consider the matrix element

$$
\left\langle\tau_{\lambda} e_{k}, \tau_{\mu} e_{l}\right\rangle=\left\langle\tau_{\lambda}\left(e_{k_{1}} \otimes \cdots \otimes e_{k_{n}}\right), \tau_{\mu}\left(e_{l_{1}} \otimes \cdots \otimes e_{l_{n}}\right)\right\rangle_{\otimes_{i=1}^{n} s_{q}^{v_{i}}},
$$

which, by Lemma 3.1, equals

$$
\begin{aligned}
& \sum_{j_{1}, \ldots, j_{n}=0}^{\infty} \frac{[-k]_{j}[-l]_{j}}{[j] ![v]_{j}} q^{(1 / 4)\{v+k+l, v+2 j\}} \lambda^{|k|-|j|} \mu^{|l|-|j|} \\
& \quad=q^{(1 / 4)\{k+l, v\}} \lambda^{|k|} \mu^{|l|} \prod_{j=1}^{n}{ }_{2} \phi_{1}\left[\begin{array}{c}
q^{-k_{j}}, q^{-l_{j}} \\
q^{v_{j}}
\end{array} ; q, \frac{q^{(1 / 2)\left(v_{j}+k_{j}+l_{j}+1-v_{j}^{*}-k_{j}^{*}-l_{j}^{*}\right)}}{\lambda \mu}\right] .
\end{aligned}
$$

Here we use the quantum algebra approach [8], considering $q$-exponentials of quantum algebra elements as generalized group elements. We remark that we would have obtained the same results considering instead the matrix elements

$$
\left\langle e_{q}\left(-\lambda X_{+} K\right) e_{k}, E_{q}\left(-\mu X_{+} K^{-1}\right) e_{l}\right\rangle_{\bigotimes_{i=1}^{n} s_{q}^{v_{i}}}
$$

where $e_{q}$ and $E_{q}$ are the $q$-exponential functions [10]

$$
e_{q}(z)=\sum_{n=0}^{\infty} \frac{z^{n}}{(q ; q)_{n}}, \quad E_{q}(z)=\sum_{n=0}^{\infty} \frac{q^{\left(\begin{array}{c}
n \\
2
\end{array}\right)} z^{n}}{(q ; q)_{n}}
$$

confer [9, 14, 44].

Consider the identity

$$
\left\langle\tau_{\lambda} \mathscr{F}_{Q}^{*} e_{k}, \tau_{\mu} e_{l}\right\rangle=\sum_{m_{1}, \ldots, m_{n}=0}^{\infty} \frac{\left[v_{1}\right]_{m_{1}} \cdots\left[v_{n}\right]_{m_{n}}}{\left[m_{1}\right] ! \cdots\left[m_{n}\right] !}\left\langle\mathscr{F}_{Q}^{*} e_{k}, e_{m}\right\rangle\left\langle\tau_{\lambda} e_{m}, \tau_{\mu} e_{l}\right\rangle .
$$


Since $\tau_{\lambda}$ is a lowering operator there is no problem with convergence. Since $\tau_{\lambda}$ commutes with $\mathscr{F}_{Q}^{*}$, the left-hand side equals

$$
T Q(l)\left\langle\tau_{\lambda} e_{k}, \tau_{\mu} e_{|l|-s}\right\rangle_{s_{q}^{|v|+2 s}}
$$

Inserting the expression (6.5) for matrix elements and letting $c=1 / \lambda \mu$ we obtain the following proposition.

Proposition 6.1. For $Q$ a highest weight vector in $\bigotimes_{i=1}^{n} A_{q}^{v_{i}}$ of degree $s$, and for $k$ a nonnegative integer, one has the identity

$$
\begin{aligned}
& \frac{[|v|+2 s]_{k}}{[k] !} T Q(l) c^{s}{ }_{2} \phi_{1}\left[\begin{array}{c}
q^{-k}, q^{s-|l|} \\
q^{|v|+2 s} ; q, q^{(1 / 2)(|v|+k+|l|+s+1)} c
\end{array}\right] \\
& \quad=\sum_{|m|=k+s} \frac{[v]_{m}}{[m] !} q^{(1 / 4)\{m+l, v\}} T Q(m) \prod_{j=1}^{n} \phi_{1}\left[\begin{array}{c}
q^{-m_{j}}, q^{-l_{j}} \\
q^{v_{j}}
\end{array} ;, q^{(1 / 2)\left(v_{j}+m_{j}+l_{j}+1-v_{j}^{*}-m_{j}^{*}-l_{j}^{*}\right)} c\right] .
\end{aligned}
$$

For $n=2$ this is a degenerate case of identities proved in [20]. We must point out that it is possible to deduce the general case from the case $n=2$ by choosing a basis in the space of highest weight vectors constructed by binary coupling; confer Section 7 . We also remark that for $\lambda=0,(6.8)$ takes the form

$$
\frac{[s-|l|]_{k}}{[k] !} T Q(l)=(-1)^{s} \sum_{|m|=k+s} \frac{[-l]_{m}}{[m] !} q^{(1 / 8)\{v+2 l, v+2 m\}} T Q(m) .
$$

A linearization formula may be obtained by considering the identity

$$
\left\langle\tau_{\lambda} e_{k}, \tau_{\mu} e_{l}\right\rangle=\sum_{s=0}^{\infty}\left\langle\Pi_{s} \tau_{\lambda} e_{k}, \tau_{\mu} e_{l}\right\rangle=\sum_{s=0}^{\min (|k|,|l|)} P_{s}(k, l)\left\langle\tau_{\lambda} e_{|k|-s}, \tau_{\mu} e_{|l|-s}\right\rangle_{s q_{q}|v|+2 s} .
$$

This leads to the following proposition.

Proposition 6.2. There is the identity

$$
\begin{gathered}
q^{(1 / 4)\{k+l, v\}} \prod_{j=1}^{n}{ }_{2} \phi_{1}\left[\begin{array}{c}
q^{-k_{j}}, q^{-l_{j}} \\
q^{v_{j}}
\end{array} ;, q^{(1 / 2)\left(v_{j}+k_{j}+l_{j}+1-v_{j}^{*}-k_{j}^{*}-l_{j}^{*}\right)} c\right] \\
=\sum_{s=0}^{\min (|k|,|l|)} P_{s}(k, l) c^{s}{ }_{2} \phi_{1}\left[\begin{array}{c}
q^{s-|k|}, q^{s-|l|} \\
q^{|v|+2 s}
\end{array} ; q, q^{(1 / 2)(|v|+|k|+|l|+1)} c\right] .
\end{gathered}
$$

Inserting the expression for coupling kernels given in Theorem 5.1, this is not hard to prove directly. It can be obtained as a special case of Verma's expansion formula [40], confer also [36]. This gives an alternative proof of Theorem 5.1, since the expansion (6.13) determines the coefficients $P_{s}$. For $n=2$, factoring $P_{s}$ as a product of two Clebsch-Gordan coefficients, this linearization formula occurs in [14, 34, 39] in a similar context. 
The special case $\lambda=0$ of (6.12) is

$$
\frac{[-l]_{k}}{[v]_{k}} q^{(1 / 8)\{v+2 l, v+2 k\}}=(-1)^{s} \sum_{s=0}^{\min (|k|,|l|)} \frac{[s-|l|]_{|k|-s}}{[|v|+2 s]_{|k|-s}} P_{s}(k, l),
$$

which, for $|l|=|k|$, reduces to

$$
\frac{[k] !}{[v]_{k}} \delta_{k, l}=\sum_{s=0}^{|k|} \frac{[|k|-s] !}{[|v|+2 s]_{|k|-s}} P_{s}(k, l) .
$$

For $n=2$, factoring $P_{s}$ as a product of two terminating ${ }_{3} \phi_{2}$ series, this is the orthogonality relation for dual $q$-Hahn polynomials.

7. Examples. In this section we will consider some examples of orthogonal and biorthogonal multivariable Hahn polynomials which may be obtained by our method.

One way to construct orthogonal bases in the space of coupling coefficients is by binary coupling. This leads to multivariable polynomials which may be factored as products of $q$-Hahn polynomials. For $q$ a power of a prime, such polynomials arise in connection with finite fields [6]. In the limit $q \rightarrow 1$, the corresponding polynomials are of importance in the quantum theory of angular momentum, and they have also been used in certain stochastic models in genetics [15]. To obtain them as coupling coefficients, we first note that if $Q_{1} \in \bigotimes_{i=1}^{k} \mathscr{A}_{q}^{v_{i}}$ and $Q_{2} \in \bigotimes_{i=k+1}^{n} \mathscr{A}_{q}^{v_{i}}$ are highest weight vectors of degrees $t_{1}, t_{2}$ and weights $-\mu_{1},-\mu_{2}$, respectively, and $Q_{s}$ is a highest weight vector of degree $s$ in $\mathscr{A}_{q}^{\mu_{1}} \otimes \mathscr{A}_{q}^{\mu_{2}}$ (unique up to a multiplicative constant), then the equation

$$
\mathscr{F}_{\left(Q_{1}, Q_{2}\right)_{s}}\left(f_{1} \otimes \cdots \otimes f_{n}\right)=\mathscr{F}_{Q_{s}}\left(\mathscr{F}_{Q_{1}}\left(f_{1} \otimes \cdots \otimes f_{k}\right) \otimes \mathscr{F}_{Q_{2}}\left(f_{k+1} \otimes \cdots \otimes f_{n}\right)\right)
$$

determines a highest weight vector $\left(Q_{1}, Q_{2}\right)_{s} \in \bigotimes_{i=1}^{n} A_{q}^{v_{i}}$. Choosing $f_{i}(z)=z^{m_{i}}$ gives

$$
T\left(Q_{1}, Q_{2}\right)_{s}(m)=T Q_{1}\left(m^{\prime}\right) T Q_{2}\left(m^{\prime \prime}\right) T Q_{s}\left(\left|m^{\prime}\right|-t_{1},\left|m^{\prime \prime}\right|-t_{2}\right),
$$

where $m^{\prime}=\left(m_{1}, \ldots, m_{k}\right), m^{\prime \prime}=\left(m_{k+1}, \ldots, m_{n}\right)$. Moreover, one has the identity

$$
\left\langle\left(Q_{1}, Q_{2}\right)_{s},\left(Q_{3}, Q_{4}\right)_{t}\right\rangle=\delta_{s t}\left\|Q_{s}\right\|^{2}\left\langle Q_{1}, Q_{3}\right\rangle\left\langle Q_{2}, Q_{4}\right\rangle
$$

As an example, the polynomials

$$
Q_{q r}=\left((1,1)_{q}, 1\right)_{r}, \quad q+r=s
$$

form an orthogonal basis in the space of highest weight vectors of degree $s$ in a threefold tensor product $\mathscr{A}_{q}^{v_{1}} \otimes \mathscr{A}_{q}^{\nu_{2}} \otimes \mathscr{A}_{q}^{\nu_{3}}$. Applying the transform $T$ gives a system of orthogonal polynomials which, by (7.2), factor as products of two $q$-Hahn polynomials. The orthogonality relation, as well as the convolution formula in Proposition 6.1, then follow from the corresponding identities for $q$-Hahn polynomials. The coefficients

$$
\left\langle\left((1,1)_{q}, 1\right)_{r},\left(1,(1,1)_{t}\right)_{u}\right\rangle
$$


are Racah coefficients, or Wigner $6 j$-symbols, for $\boldsymbol{q}_{q^{1 / 2}}(\mathfrak{s u}(1,1))$, which may be identified with $q$-Racah polynomials [16]. For an $(n+1)$-fold tensor product, there is a large number of ways to construct orthogonal bases by binary coupling, and the coefficients for a change between two such bases are given by Wigner $3 n j$-symbols.

As a different example of coupling coefficients, we will construct $q$-analogues of certain biorthogonal multivariable Hahn polynomials introduced by Rahman [27] for $n=3$ and by Tratnik [38] in general. For Jacobi-type weights, this kind of polynomials were first studied by Appell; confer [1]. In the case $q=1$, they correspond to highest weight vectors of the form

$$
\left(z_{1}-z_{n}\right)^{t_{1}} \cdots\left(z_{n-1}-z_{n}\right)^{t_{n-1}}
$$

and to the dual basis.

It will be convenient to write $\mathscr{D}_{q}$ (for Dirichlet, cf. Appendix A) for the space of polynomials in one variable, viewed as a $U$-module with the action

$$
X_{+} z^{k}=-[k] z^{k-1}, \quad X_{-} z^{k}=[k] z^{k+1}, \quad K^{ \pm} z^{k}=q^{\mp(1 / 2) k} z^{k},
$$

which may be obtained by formally letting $v=0$ in (2.28). Write $\delta$ for the dilation operator $\delta f(z)=f(q z)$, so that $K=q^{-v / 4} \delta^{-1 / 2}$ as an operator on $\mathscr{A}_{q}^{v}$. Thus

$$
-\left.X_{+}\right|_{\otimes_{i=1}^{n} s q_{q}^{v_{i}}}=q^{(1 / 4) v_{1}^{*}} D_{q} \otimes \delta^{-1 / 2} \otimes \cdots \otimes \delta^{-1 / 2}+\cdots+q^{(1 / 4) v_{n}^{*}} \delta^{1 / 2} \otimes \cdots \otimes \delta^{1 / 2} \otimes D_{q},
$$

where $D_{q}$ is the $q$-derivative (2.9), while

$$
-\left.X_{+}\right|_{\mathscr{D}_{q}^{\otimes n}}=D_{q} \otimes \delta^{-1 / 2} \otimes \cdots \otimes \delta^{-1 / 2}+\cdots+\delta^{1 / 2} \otimes \cdots \otimes \delta^{1 / 2} \otimes D_{q}
$$

Applying the commutation rule

$$
D_{q} \delta^{\lambda}=q^{\lambda} \delta^{\lambda} D_{q}
$$

gives the following lemma.

LEMMA 7.1. The operators $\left.X_{+}\right|_{\bigotimes_{i=1}^{n} s_{q}^{v_{i}}}$ and $\left.X_{+}\right|_{\mathscr{Q}_{q}^{\otimes n}}$ are connected by

$$
\left.X_{+}\right|_{\bigotimes_{i=1}^{n} s_{q}^{v_{i}}}=\left.S^{-1} X_{+}\right|_{\mathscr{D}_{q}^{\otimes n}} S,
$$

where

$$
S=\delta^{(1 / 4) v_{1}^{*}} \otimes \cdots \otimes \delta^{(1 / 4) v_{n}^{*}} .
$$

In particular, this shows that if $\mathscr{V}_{s}$ is the space of highest weight vectors of degree $s$ in $\bigotimes_{i=1}^{n} \mathcal{A}_{q}^{v_{i}}$, then $S \mathscr{V}_{s}$ is, as a space of polynomials, independent of the parameters $v_{i}$.

LEMMA 7.2. Let $j$ be an integer with $1 \leq j \leq n$. If $f$ and $g$ are two elements in $\mathscr{D}_{q}^{\otimes n}$ such that $f$ depends only on the variables $z_{1}, \ldots, z_{j}$ and $g$ only on $z_{j}, \ldots, z_{n}$, then

$$
X_{+}(f g)=X_{+} f K g+K^{-1} f X_{+} g
$$


Proof. For $n=1$ this is (2.10). To prove it in general, decompose $\mathscr{D}_{q}^{\otimes n}$ as $\mathscr{D}_{q}^{\otimes(j-1)} \otimes$ $\mathscr{D}_{\mathcal{q}} \otimes \mathscr{D}_{\mathcal{q}}^{\otimes(n-j)}$. By linearity, one may assume $f=f_{1} \otimes f_{2} \otimes 1, g=1 \otimes g_{2} \otimes g_{3}$. The lemma then follows from the case $n=1$, using the coproduct rule

$$
X_{+}=X_{+} \otimes K \otimes K+K^{-1} \otimes X_{+} \otimes K+K^{-1} \otimes K^{-1} \otimes X_{+} .
$$

The lemma also follows from the results of Appendix B, where we describe a product - on $\mathscr{D}_{q}^{\otimes n}$ which satisfies

$$
X_{+}(f \bullet g)=X_{+} f \bullet K g+K^{-1} f \bullet X_{+} g
$$

and which agrees with the usual product of polynomials under the assumptions of the lemma.

Proposition 7.3. For any multi-index $t=\left(t_{1}, \ldots, t_{n-1}\right)$, the polynomial

$$
\begin{aligned}
\tilde{Q}_{t}(z)= & \prod_{j=0}^{t_{1}-1}\left(z_{1}-q^{-\left(j+t_{2}+\cdots+t_{n-1}\right)} z_{n}\right) \\
& \times \prod_{j=0}^{t_{2}-1}\left(z_{2}-q^{-\left(j+t_{3}+\cdots+t_{n-1}\right)} z_{n}\right) \cdots \prod_{j=0}^{t_{n-1}-1}\left(z_{n-1}-q^{-j} z_{n}\right)
\end{aligned}
$$

is annihilated by $\left.X_{+}\right|_{\mathscr{D}_{q}^{\otimes n}}$, and thus

$$
\begin{aligned}
Q_{t}(z) & =q^{(1 / 4)\left(v_{1}^{*} t_{1}+\cdots+v_{n-1}^{*} t_{n-1}\right)} S^{-1} \tilde{Q}_{t}(z) \\
& =q^{(1 / 4)\{(t, 0), v\}} \tilde{Q}_{t}\left(q^{-(1 / 4) v_{1}^{*}} z_{1}, \ldots, q^{-(1 / 4) v_{n}^{*}} z_{n}\right)
\end{aligned}
$$

is a highest weight vector in $\bigotimes_{i=1}^{n} \mathscr{A}_{q}^{v_{i}}$.

Note that

$$
\left.Q_{t}\right|_{z_{n}=0}=z_{1}^{t_{1}} \cdots z_{n-1}^{t_{n-1}}
$$

Since the dimension of the space of monomials of degree $s$ in $n-1$ variables equals the dimension $\left(\begin{array}{c}n+s-2 \\ n-2\end{array}\right)$ of the space of highest weight vectors of degree $s$, the polynomials $\left(Q_{t}\right)_{|t|=s}$ form a basis of the latter space. In particular, it follows that $Q_{t}$ is the unique highest weight vector satisfying (7.18).

Proof of Proposition 7.3. Let $j$ be the smallest number such that $t_{j} \neq 0$. Then

$$
\tilde{Q}_{t}\left(z_{1}, \ldots, z_{n}\right)=\left(z_{j}-q^{1-|t|} z_{n}\right) \tilde{Q}_{t^{\prime}}\left(z_{1}, \ldots, z_{n}\right),
$$

where $t^{\prime}=\left(0, \ldots, 0, t_{j}-1, t_{j+1}, \ldots, t_{n}\right)$. Proceeding by induction on $|t|$, we may assume that $X_{+} \tilde{Q}_{t^{\prime}}=0$. Since $\tilde{Q}_{t^{\prime}}$ depends only on the variables $z_{j}, \ldots, z_{n}$, Lemma 7.2 gives

$$
\begin{aligned}
X_{+} \tilde{Q}_{t} & =X_{+}\left(z_{j} \tilde{Q}_{t^{\prime}}\right)-q^{1-|t|} X_{+}\left(\tilde{Q}_{t^{\prime}} z_{n}\right) \\
& =X_{+} z_{j} K \tilde{Q}_{t^{\prime}}+K^{-1} z_{j} X_{+} \tilde{Q}_{t^{\prime}}-q^{1-|t|} X_{+} \tilde{Q}_{t^{\prime}} K z_{n}-q^{1-|t|} K^{-1} \tilde{Q}_{t^{\prime}} X_{+} z_{n} .
\end{aligned}
$$

Since $X_{+} \tilde{Q}_{t^{\prime}}=0, X_{+} z_{j}=X_{+} z_{n}=-1$ and $K^{ \pm} \tilde{Q}_{t^{\prime}}=q^{\mp(1 / 2)(|t|-1)} \tilde{Q}_{t^{\prime}}$, it follows that $X_{+} \tilde{Q}_{t}=0$. 
To obtain an expression for the coupling coefficients $P_{t}=T Q_{t}$, we expand $Q$ into monomials. Using the terminating $q$-binomial formula

$$
\prod_{j=0}^{s-1}\left(x-q^{-j} y\right)=(-1)^{s} \sum_{l=0}^{s} \frac{[-s]_{l}}{[l] !} q^{-(1 / 2)(s-l)(s-1)} x^{l} y^{s-l},
$$

one obtains

$$
\begin{aligned}
\tilde{Q}_{t}(z)=(-1)^{s} \sum_{l_{1}, \ldots, l_{n-1}} \frac{\left[-t_{1}\right]_{l_{1}} \cdots\left[-t_{n-1}\right]_{l_{n-1}}}{\left[l_{1}\right] ! \cdots\left[l_{n-1}\right] !} \\
\quad \times q^{-(1 / 2) \sum_{i}\left(t_{i}-l_{i}\right)\left(t_{i}-1\right)-\sum_{i<j}\left(t_{i}-l_{i}\right) t_{j}} z_{1}^{l_{1}} \cdots z_{n-1}^{l_{n-1}} z_{n}^{s-l_{1}-\cdots-l_{n-1}},
\end{aligned}
$$

where $s=|t|$. Put $l_{n}=s-l_{1}-\cdots-l_{n-1}$. Using (4.8) one may then rewrite the exponent of $q$ as

$$
\frac{1}{2} l_{n}+\frac{1}{2}\{(t, 0), l\}
$$

Writing also

$$
\frac{1}{2}\{(t, 0), l\}+\frac{1}{4}\{(t, 0), v\}+\frac{1}{4}\{v, l\}=\frac{1}{8}\{v+2(t, 0), v+2 l\}
$$

gives

$$
Q_{t}(z)=(-1)^{s} \sum_{l_{1}+\cdots+l_{n}=s} \frac{\left[-t_{1}\right]_{l_{1}} \cdots\left[-t_{n-1}\right]_{l_{n-1}}}{\left[l_{1}\right] ! \cdots\left[l_{n-1}\right] !} q^{(1 / 2) l_{n}+(1 / 8)\{v+2(t, 0), v+2 l\}} z_{1}^{l_{1}} \cdots z_{n}^{l_{n}}
$$

and thus

$$
\begin{aligned}
P_{t}(m)=\sum_{l_{1}+\cdots+l_{n}=s} & \frac{\left[-t_{1}\right]_{l_{1}} \cdots\left[-t_{n-1}\right]_{l_{n-1}}}{\left[l_{1}\right] ! \cdots\left[l_{n-1}\right] !} \\
& \times \frac{\left[-m_{1}\right]_{l_{1}} \cdots\left[-m_{n}\right]_{l_{n}}}{\left[v_{1}\right]_{l_{1}} \cdots\left[v_{n}\right]_{l_{n}}} q^{(1 / 2) l_{n}+(1 / 4)\{v+m+(t, 0), v+2 l\}} .
\end{aligned}
$$

Next we consider the dual basis of $\left(P_{t}\right)_{|t|=s}$. It can be expressed in terms of coupling kernels. To see this we first observe that, because of (7.18),

$$
\left\langle Q_{t}, z_{1}^{u_{1}} \cdots z_{n-1}^{u_{n-1}}\right\rangle=\frac{\left[t_{1}\right] ! \cdots\left[t_{n-1}\right] !}{\left[v_{1}\right]_{t_{1}} \cdots\left[v_{n-1}\right]_{t_{n-1}}} \delta_{t, u}
$$

for arbitrary $u_{i}$. Thus the dual basis $\left(Q_{t}^{\prime}\right)$ of $\left(Q_{t}\right)$ is given by

$$
Q_{t}^{\prime}=\frac{\left[v_{1}\right]_{t_{1}} \cdots\left[v_{n-1}\right]_{t_{n-1}}}{\left[t_{1}\right] ! \cdots\left[t_{n-1}\right] !} \Pi_{s}\left(z_{1}^{t_{1}} \cdots z_{n-1}^{t_{n-1}}\right),
$$

where, as above, $\Pi_{s}$ is the orthogonal projection onto the space of highest weight 
vectors. It follows from (5.9) that, in general,

$$
T \Pi_{s}\left(z_{1}^{t_{1}} \cdots z_{n}^{t_{n}}\right)(m)=P_{s}(t, m), \quad|t|=s,
$$

which gives

$$
P_{t}^{\prime}(m)=T Q_{t}^{\prime}(m)=\frac{\left[v_{1}\right]_{t_{1}} \cdots\left[v_{n-1}\right]_{t_{n-1}}}{\left[t_{1}\right] ! \cdots\left[t_{n-1}\right] !} P_{s}\left(t_{1}, \ldots, t_{n-1}, 0, m_{1}, \ldots, m_{n}\right) .
$$

We insert the explicit expression for $P_{s}$ from Theorem 5.1. Then the summation variable $j_{n}$ may be put equal to zero. Writing $\hat{x}=\left(x_{1}, \ldots, x_{n-1}\right)$ for $x \in \mathbb{R}^{n}$, we obtain

$$
\begin{aligned}
P_{t}^{\prime}(m)= & \frac{[-|m|]_{s}}{[|v|+s-1]_{s}} \frac{[\hat{v}]_{t}}{[t] !} \\
& \times \sum_{j_{1}, \ldots, j_{n-1}=0}^{\infty} \frac{[|v|+s-1]_{|j|}}{[-|m|]_{|j|}} \frac{[-t]_{j}[-\hat{m}]_{j}}{[\hat{v}]_{j}[j] !} q^{(1 / 4)\{v+m+(t, 0), v+2(j, 0)\}}
\end{aligned}
$$

(which should be interpreted as a finite sum, as in Theorem 5.1). Thus, as a consequence of Theorem 4.1, we have the following fact.

THEOREM 7.4. The coupling coefficients $P_{t}(m)$ and $P_{u}^{\prime}(m)$ given by (7.26) and (7.31), respectively, satisfy the relations

$$
\sum_{|m|=s+k} \frac{\left[v_{1}\right]_{m_{1}} \cdots\left[v_{n}\right]_{m_{n}}}{\left[m_{1}\right] ! \cdots\left[m_{n}\right] !} P_{t}(m) P_{u}^{\prime}(m)=\frac{[|v|+2 s]_{k}}{[k] !} \delta_{t, u},
$$

where $s=|t|($ or $s=|u|)$.

As indicated in Section 4, we may view this as a biorthogonality relation for a complete system of $(n-1)$-variable polynomials. It may be worth writing this out explicitly, in standard notation. To facilitate comparison with the one-variable case, we view the $q^{v_{i}-1}$ as parameters and normalize the polynomials so that they take the value 1 at $m_{1}=\cdots=m_{n-1}=0$. Thus, we write

$$
\begin{aligned}
& \frac{\mathcal{q}^{-(1 / 2)|t||m|-(1 / 4)\{m, v\}} P_{t}(m)}{\mathcal{q}^{-(1 / 2)|t||m|-(1 / 4)|m||\hat{v}|} P_{t}(0,|m|)} \\
& \quad=p_{t}\left(q^{-m_{1}}, q^{-\left(m_{1}+m_{2}\right)}, \ldots, q^{-\left(m_{1}+\cdots+m_{n-1}\right)} ; q^{v_{1}-1}, \ldots, q^{v_{n}-1},|m| ; q\right),
\end{aligned}
$$

and $p_{t}^{\prime}$ for the polynomials similarly obtained from $P_{t}^{\prime}$. Replacing $n$ by $n+1$, and writing $\Phi_{1: 1}^{1: 2}$ for the $q$-Kampé de Fériet function

$$
\begin{aligned}
\Phi_{1: 1}^{1: 2}\left(\begin{array}{c}
a \\
d
\end{array}: \begin{array}{c}
b_{1}, c_{1} \\
e_{1}
\end{array} ; \ldots ; \begin{array}{c}
b_{n}, c_{n} \\
e_{n}
\end{array} ; q ; x_{1}, \ldots, x_{n}\right) \\
\quad=\sum_{k_{1}, \ldots, k_{n}=0}^{\infty} \frac{(a ; q)_{|k|}}{(d ; q)_{|k|}} \frac{\left(b_{1} ; q\right)_{k_{1}}\left(c_{1} ; q\right)_{k_{1}}}{(q ; q)_{k_{1}}\left(e_{1} ; q\right)_{k_{1}}} \cdots \frac{\left(b_{n} ; q\right)_{k_{n}}\left(c_{n} ; q\right)_{k_{n}}}{(q ; q)_{k_{n}}\left(e_{n} ; q\right)_{k_{n}}} x_{1}^{k_{1}} \cdots x_{n}^{k_{n}},
\end{aligned}
$$


we have

$$
\begin{aligned}
& p_{t}\left(x_{1}, \ldots, x_{n} ; a_{1}, \ldots, a_{n}, b, N ; q\right) \\
& =x_{n}^{|t|} \frac{\left(x_{n}^{-1} q^{-N} ; q\right)_{|t|}}{\left(q^{-N} ; q\right)_{|t|}} \Phi_{1: 1}^{1: 2}\left(\begin{array}{c}
b^{-1} q^{-|t|} \\
x_{n} q^{1+N-|t|}
\end{array}: \begin{array}{c}
q^{-t_{1}}, x_{1} \\
a_{1} q
\end{array} ; \begin{array}{c}
q^{-t_{2}}, x_{2} / x_{1} \\
a_{2} q
\end{array} ; \ldots ; \begin{array}{c}
q^{-t_{n}}, x_{n} / x_{n-1} \\
a_{n} q
\end{array}\right. \\
& q ; a_{1} \cdots a_{n} b q^{N+|t|+n+1}, a_{2} \cdots a_{n} b x_{1} q^{N+|t|-t_{1}+n} \\
& \left., \ldots, a_{n} b x_{n-1} q^{N+t_{n}+2}\right), \\
& p_{t}^{\prime}\left(x_{1}, \ldots, x_{n} ; a_{1}, \ldots, a_{n}, b, N ; q\right)
\end{aligned}
$$

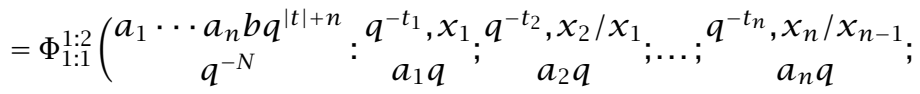

$$
\begin{aligned}
& \left.q ; q, \frac{x_{1}}{a_{1}} q^{-t_{1}}, \ldots, \frac{x_{n-1}}{a_{1} \cdots a_{n-1}} q^{2-n-|t|+t_{n}}\right) .
\end{aligned}
$$

In terms of these polynomials, Theorem 7.4 may be rewritten as

$$
\begin{aligned}
& \sum_{0 \leq m_{1} \leq \cdots \leq m_{n} \leq N} \frac{\left(a_{1} q ; q\right)_{m_{1}}\left(a_{2} q ; q\right)_{m_{2}-m_{1}} \cdots\left(a_{n} q ; q\right)_{m_{n}-m_{n-1}}(b q ; q)_{N-m_{n}}}{(q ; q)_{m_{1}}(q ; q)_{m_{2}-m_{1}} \cdots(q ; q)_{m_{n}-m_{n-1}}(q ; q)_{N-m_{n}}} \prod_{j=1}^{n}\left(a_{j} q\right)^{-m_{j}} \\
& \times p_{t}\left(q^{-m_{1}}, \ldots, q^{-m_{n}} ; a_{1}, \ldots, a_{n}, b, N ; q\right) p_{u}^{\prime}\left(q^{-m_{1}}, \ldots, q^{-m_{n}} ; a_{1}, \ldots, a_{n}, b, N ; q\right) \\
& =\delta_{t u} \frac{(q ; q)_{t_{1}} \cdots(q ; q)_{t_{n}}}{\left(a_{1} q ; q\right)_{t_{1}} \cdots\left(a_{n} q ; q\right)_{t_{n}}} \frac{(b q ; q)_{|t|}}{\left(q^{-N} ; q\right)_{|t|}} \frac{\left(a_{1} \cdots a_{n} b q^{n+|t|} ; q\right)_{N+1}}{(q ; q)_{N}} \\
& \times \frac{1}{1-a_{1} \cdots a_{n} b q^{n+2|t|}}(-1)^{|t|} q^{\left(\begin{array}{c}
|t| \\
2
\end{array}\right)-N|t|}\left(a_{1} \cdots a_{n} q^{n}\right)^{-N} \prod_{1 \leq i \leq j \leq n}\left(a_{j} q\right)^{t_{i}} .
\end{aligned}
$$

\section{Appendices}

A. Quasi-commuting homogeneous coordinates. In this appendix we will use quasi-commuting homogeneous coordinates to give a "geometric" motivation for the holomorphic realization, where geometric may be understood in the sense of noncommutative geometry (cf. [25]). First recall the classical case. If $f$ is a function defined in the unit disc, one may (at least for integer $v$ ) introduce a function $F$ defined in the cone $|x|<|y|$ in $\mathbb{C}^{2}$ by

$$
F(x, y)=\frac{1}{y^{v}} f\left(\frac{x}{y}\right)
$$

The natural right action on $F$ by SU(1,1),

$$
F(x, y) \longmapsto F(a x+b y, c x+d y), \quad\left(\begin{array}{ll}
a & b \\
c & d
\end{array}\right) \in \mathrm{SU}(1,1)
$$

then gives rise to the action (1.3) on $f$. 
In the quantum case we must replace the coordinate functions $x, y$ by quasicommuting elements, that is, quantities satisfying $x y=q^{1 / 2} y x$. Since we need fractional powers, we let $\mathscr{F}_{q}$ be the associative algebra over $\mathbb{C}$ generated by $x^{\alpha}, y^{\alpha}, \alpha \in \mathbb{R}$, with relations

$$
x^{0}=y^{0}=1, \quad x^{\alpha} x^{\beta}=x^{\alpha+\beta}, \quad y^{\alpha} y^{\beta}=y^{\alpha+\beta}, \quad x^{\alpha} y^{\beta}=q^{(1 / 2) \alpha \beta} y^{\beta} x^{\alpha} .
$$

To proceed, we need a $q$-analogue of the action of $\operatorname{SL}(2, \mathbb{C})$ on functions on $\mathbb{C}^{2}$. This will be the action of $u$ on $\mathscr{F}_{q}$ given by

$$
\begin{aligned}
& X_{+}\left(x^{\alpha} y^{\beta}\right)=-q^{(1 / 4)(1+\beta-\alpha)}[\alpha] x^{\alpha-1} y^{\beta+1}, \\
& X_{-}\left(x^{\alpha} y^{\beta}\right)=-q^{(1 / 4)(1+\alpha-\beta)}[\beta] x^{\alpha+1} y^{\beta-1}, \\
& K^{ \pm}\left(x^{\alpha} y^{\beta}\right)=q^{ \pm(1 / 4)(\beta-\alpha)} x^{\alpha} y^{\beta} .
\end{aligned}
$$

In fact, this makes $\mathscr{F}_{q}$ into a $U$-module algebra, that is, the equations

$$
\begin{aligned}
K^{ \pm}(1) & =1, \quad X_{ \pm}(1)=0, \\
K^{ \pm}(f g) & =K^{ \pm}(f) K^{ \pm}(g), \\
X_{ \pm}(f g) & =X_{ \pm}(f) K(g)+K^{-1}(f) X_{ \pm}(g),
\end{aligned}
$$

are satisfied. The existence of this module algebra is well known.

We now fix $v>0$, consider the $U$-submodule of $\mathscr{F}_{q}$ generated by $\left(x^{k} y^{-v-k}\right)_{k=0}^{\infty}$, pass to the inhomogeneous notation

$$
z^{k}=q^{(1 / 4) k(v+k)} x^{k} y^{-v-k}=q^{-(1 / 4) k(v+k)} y^{-v-k} x^{k}
$$

and identify the $z^{k}$ with powers of a complex variable. The action (A.4) is then given by

$$
\begin{aligned}
& X_{+} z^{k}=-[k] z^{k-1}, \\
& X_{-} z^{k}=[v+k] z^{k+1}, \\
& K^{ \pm} z^{k}=q^{\mp(1 / 4)(v+2 k)} z^{k},
\end{aligned}
$$

or equivalently by (2.28). If this defines a unitary action on a Hilbert space in the sense of (2.19), it is easily seen that one must have (assuming the normalizing condition $\|1\|=1)$

$$
\left\|z^{k}\right\|^{2}=\frac{[k] !}{[v]_{k}} .
$$

Thus we recover the unitary representation of $U$ on the space $\mathscr{A}_{q}^{v}$.

Similarly, for $v=0$ we obtain the space $\mathscr{D}_{q}$ considered in Section 7 . Since

$$
q^{(1 / 4) k^{2}} x^{k} y^{-k}=\left(q^{(1 / 4)} x y^{-1}\right)^{k}
$$

the map $z^{k} \mapsto q^{k^{2} / 4} x^{k} y^{-k}$ embeds $\mathscr{D}_{q}$ as a sub-module algebra of $\mathscr{F}_{q}$. We will exploit this fact in the next appendix. We also mention that $\mathscr{D}_{\mathfrak{q}} / \mathbb{C}$ is a unitarizable 


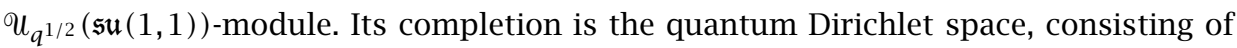
analytic functions on the disc $|z|<q^{-1 / 2}$, modulo constants, with the norm

$$
\|f\|^{2}=\sum_{k=1}^{\infty}[k]|\hat{f}(k)|^{2}=\left\|D_{q} f\right\|_{\mathscr{A}_{q}^{2}}^{2} .
$$

It is equivalent to the quantum Bergman space $\mathscr{A}_{q}^{2}$, an intertwining map being the $q$-derivative $D_{q}$.

B. The algebra of highest weight vectors. In this section we will describe the kernel of $X_{+}$in $\otimes_{i=1}^{n} A_{q}^{v_{i}}$. In the case $q=1, X_{+}=-\sum_{i=1}^{n} \partial / \partial z_{i}$ satisfies the Leibniz rule $X_{+}(f g)=X_{+}(f) g+f X_{+}(g)$, so the kernel is formally an associative algebra. (The product is unbounded, but one can obtain a well-defined algebra for instance as the subspace of polynomials.) In this appendix we will find a product $\bullet$ on the space of polynomials in $\bigotimes_{i=1}^{n} \mathscr{A}_{q}^{v_{i}}$ which satisfies

$$
X_{+}(f \bullet g)=X_{+}(f) \bullet K(g)+K^{-1}(f) \bullet X_{+}(g),
$$

so that it gives an algebra structure to the space of polynomials annihilated by $X_{+}$, that is, to the span of the highest weight vectors.

First we recall the universal $R$-matrix

$$
R=q^{(1 / 4)(H \otimes H)} \sum_{j=0}^{\infty} \frac{\left(1-q^{-1}\right)^{j}}{[j] !} q^{(1 / 4) j(j-1)}\left(K X_{+}\right)^{j} \otimes\left(K^{-1} X_{-}\right)^{j},
$$

where $H$ satisfies $q^{(1 / 4) H}=K$. It may be viewed as an element of a suitable extension of $u \otimes U$. For "nice" $U$-modules $V_{1}$ and $V_{2}$,

$$
\Psi=\sigma \circ R: V_{1} \otimes V_{2} \longrightarrow V_{2} \otimes V_{1}
$$

is intertwining, where $\sigma$ is the flip $\sigma(f \otimes g)=g \otimes f$. We will only need this when $V_{1}=V_{2}=\mathscr{D}_{q}$.

There is a canonical way to give a module algebra structure to a tensor product of module algebras, known as the braided tensor product [24]. Namely, if $A_{1}$ and $A_{2}$ are U-module algebras with products

$$
m_{i}: A_{i} \otimes A_{i} \longrightarrow A_{i}, \quad i=1,2,
$$

then $A_{1} \otimes A_{2}$ is another one with the product

$$
\left(m_{1} \otimes m_{2}\right) \circ(1 \otimes \Psi \otimes 1) .
$$

This construction is associative in the sense that regardless of how it is iterated to define a module algebra structure on a finite tensor product $A_{1} \otimes \cdots \otimes A_{n}$, the result is the same.

Consider the space $\mathscr{D}_{q} \otimes \mathscr{D}_{q}$, consisting of polynomials in two variables $z_{1}$ and $z_{2}$, and view it as a sub-module algebra of the braided tensor product $\mathscr{F}_{q} \otimes \mathscr{F}_{q}$. Thus we write

$$
z_{1}=q^{1 / 4} x y^{-1} \otimes 1, \quad z_{2}=1 \otimes q^{1 / 4} x y^{-1}
$$


Denoting by $\bullet$ the product defined by (B.5), we have

$$
z_{1} \cdot z_{2}=q^{1 / 4} x y^{-1} \otimes q^{1 / 4} x y^{-1}=z_{1} z_{2},
$$

where the product on the right-hand side is the usual product of polynomials, while

$$
\begin{aligned}
z_{2} \bullet z_{1} & =\Psi\left(q^{1 / 4} x y^{-1} \otimes q^{1 / 4} x y^{-1}\right) \\
& =q^{3 / 2} x y^{-1} \otimes x y^{-1}+(1-q) x^{2} y^{-2} \otimes 1 \\
& =q z_{1} z_{2}+(1-q) z_{1}^{2} .
\end{aligned}
$$

By associativity, this determines the product $\bullet$ uniquely. One may prove by induction that, more generally, the product on $\mathscr{D}_{q}^{\otimes n}$ considered as a sub-module algebra of $\mathscr{F}_{q}^{\otimes n}$ is determined by

$$
z_{i} \bullet z_{j}= \begin{cases}z_{i} z_{j}, & i \leq j, \\ q z_{j} z_{i}+(1-q) z_{j}^{2}, & i>j\end{cases}
$$

With this product, the kernel of $X_{+}$is a graded algebra $\left.\operatorname{Ker} X_{+}\right|_{\mathscr{P}_{q}^{\otimes n}}=\sum_{s=0}^{\infty} \tilde{\mathscr{V}}_{s}$, where $\tilde{V}_{s}$ consists of homogeneous polynomials of degree $s$. It is generated by $\tilde{\mathscr{V}}_{0}$, which is the space of constants, together with $\tilde{\mathscr{V}}_{1}=\left\{a_{1} z_{1}+\cdots+a_{n} z_{n} ; a_{1}+\cdots+a_{n}=0\right\}$.

By Lemma 7.1, these observations carry over to the space $\otimes_{i=1}^{n} A^{v_{i}}$. The span of all highest weight vectors is a graded algebra generated by 1 and $\mathscr{V}_{1}=S^{-1} \tilde{V}_{1}$, with the product

$$
Q_{1} \bullet Q_{2}=S^{-1}\left(S Q_{1} \bullet S Q_{2}\right)
$$

given on $z_{i}$ by

$$
z_{i} \bullet z_{j}= \begin{cases}z_{i} z_{j}, & i \leq j, \\ q z_{j} z_{i}+(1-q) q^{(1 / 4)\left(v_{i}^{*}-v_{j}^{*}\right)} z_{j}^{2}, & i>j,\end{cases}
$$

where $v_{i}^{*}-v_{j}^{*}=v_{j}+2\left(v_{j+1}+\cdots+v_{i-1}\right)+v_{i}$.

For $n=2$, the space $\tilde{V}_{s}$ is one-dimensional and generated by $\left(z_{1}-z_{2}\right)^{\bullet s}$. Now, by a generalized binomial formula due to Benaoum [3], (cf. also [33]), one has

$$
\left(z_{1}-z_{2}\right)^{s}=\sum_{k=0}^{s} \frac{[s] !}{[k] ![s-k] !} q^{(1 / 2) k(s-1)}(-1)^{s-k} z_{1}^{k} z_{2}^{s-k}
$$

for variables satisfying $z_{2} z_{1}=q z_{1} z_{2}+(1-q) z_{1}^{2}$. However, for commuting variables, the right-hand side of this equation equals

$$
\prod_{j=0}^{s-1}\left(q^{j} z_{1}-z_{2}\right)
$$

which thus generates $\tilde{\mathscr{V}}_{s}$. Applying $S^{-1}$ gives, up to a multiplicative constant, the polynomial (3.14). 
ACKNOwLedgement. This paper is based on a chapter of my Ph.D. thesis [32], written at Lund University. I would like to thank my thesis advisor, Professor Jaak Peetre, for many fruitful discussions.

\section{REFERENCES}

[1] P. Appell and J. Kampé de Fériet, Fonctions Hypergéométriques et HypersphériquesPolynômes d'Hermite, Gauthier-Villars, Paris, 1926 (French).

[2] R. M. Ašerova, J. F. Smirnov, and V. N. Tolstol̆, Projection operators for simple Lie groups, Teoret. Mat. Fiz. 8 (1971), no. 2, 255-271 (Russian). MR 57\#14967. Zbl 0223.22019.

[3] H. B. Benaoum, $(q, h)$-analogue of Newton's binomial formula. Addendum to: " $h$ analogue of Newton's binomial formula", J. Phys. A 32 (1999), no. 10, 2037-2040. MR 99m:05004b.

[4] V. Chari and A. Pressley, A Guide to Quantum Groups, Cambridge University Press, Cambridge, 1994. MR 95j:17010. Zbl 0839.17009.

[5] A. Clebsch, Über eine Eigenschaft der Kugelfunctionen, J. Reine Angew. Math. 60 (1862), 343-350 (German).

[6] C. F. Dunkl, Orthogonal polynomials in two variables of q-Hahn and q-Jacobi type, SIAM J. Algebraic Discrete Methods 1 (1980), no. 2, 137-151. MR 81k:33011. Zbl 0499.33007.

[7] E. B. Elliott, On inverses of $\nabla^{2}$ and other quadratic operators, Quart. J. Math. 48 (1917), 372-379.

[8] R. Floreanini and L. Vinet, On the quantum group and quantum algebra approach to q-special functions, Lett. Math. Phys. 27 (1993), no. 3, 179-190. MR 94e:33040. Zbl 0780.33012 .

[9] _,$\cup_{q}(\operatorname{sl}(2))$ and $q$-special functions, Lie Algebras, Cohomology, and New Applications to Quantum Mechanics (Springfield, MO, 1992), Contemp. Math., vol. 160, American Mathematical Society, Rhode Island, 1994, pp. 85-100. MR 95b:33054. Zbl 0807.33013.

[10] G. Gasper and M. Rahman, Basic Hypergeometric Series, Encyclopedia of Mathematics and its Applications, vol. 34, Cambridge University Press, Cambridge, 1990. MR 91d:33034. Zbl 0695.33001.

[11] Y. I. Granovskiĭ and A. S. Zhedanov, "Twisted" Clebsch-Gordan coefficients for $\mathrm{SU}_{\mathcal{q}}$ (2), J. Phys. A 25 (1992), no. 17, L1029-L1032. MR 93e:81050. Zbl 0765.17015.

[12] W. Hahn, Über Orthogonalpolynome, die q-Differenzengleichungen genügen, Math. Nachr. 2 (1949), 4-34 (German). MR 11,29b. Zbl 0031.39001.

[13] B. Jurčo, On coherent states for the simplest quantum groups, Lett. Math. Phys. 21 (1991), no. 1, 51-58. MR 92b:81079. Zbl 0735.17024.

[14] E. G. Kalnins, W. Miller Jr., and S. Mukherjee, Models of q-algebra representations: matrix elements of $U_{\mathcal{q}}\left(\mathrm{su}_{2}\right)$, Lie Algebras, Cohomology, and New Applications to Quantum Mechanics (Springfield, MO, 1992), Contemp. Math., vol. 160, American Mathematical Society, Rhode Island, 1994, pp. 191-208. MR 95b:33055. Zbl 0815.33012.

[15] S. Karlin and J. McGregor, Linear growth models with many types and multidimensional Hahn polynomials, Theory and Application of Special Functions (Proc. Advanced Sem., Math. Res. Center, Univ. Wisconsin, Madison, Wis., 1975), Math. Res. Center, Univ. Wisconsin, Publ., no. 35, Academic Press, New York, 1975, pp. 261-288. MR 53\#10361. Zbl 0361.60071.

[16] A. N. Kirillov and N. Y. Reshetikhin, Representations of the algebra $U_{q}(\operatorname{sl}(2))$, qorthogonal polynomials and invariants of links, Infinite-Dimensional Lie Algebras and Groups (Luminy-Marseille, 1988), Adv. Ser. Math. Phys., vol. 7, World Scientific, New Jersey, 1989, pp. 285-337. MR 90m:17022. Zbl 0742.17018.

[17] S. Klimek and A. Lesniewski, The two-parameter quantum deformation of the unit disc, J. Funct. Anal. 115 (1993), no. 1, 1-23. MR 94e:46128. Zbl 0780.58020. 
[18] R. Koekoek and R. F. Swarttouw, The Askey scheme of hypergeometric orthogonal polynomials and its q-analogue, report 98-17, Delft University of Technology http: //aw.twi.tudelft.nl/ ${ }^{\sim}$ koekoek/research.html, 1998.

[19] H. T. Koelink and T. H. Koornwinder, The Clebsch-Gordan coefficients for the quantum group $\mathrm{S}_{\mu} \mathrm{U}(2)$ and $q$-Hahn polynomials, Nederl. Akad. Wetensch. Indag. Math. 51 (1989), no. 4, 443-456. MR 91c:17009. Zbl 0708.22007.

[20] H. T. Koelink and J. Van der Jeugt, Convolutions for orthogonal polynomials from Lie and quantum algebra representations, SIAM J. Math. Anal. 29 (1998), no. 3, 794-822 (electronic). MR 2000i:33022.

[21] L. I. Korogodsky, Quantum G-spaces and q-oscillators, Institute for Theoretical Physics, Kiev, preprint, 1991.

[22] N. A. Liskova and A. N. Kirillov, Clebsch-Gordan and Racah-Wigner coefficients for $U_{q}(\mathrm{SU}(1,1))$, Infinite Analysis, Part A, B (Kyoto, 1991), World Scientific, New Jersey, 1992, pp. 611-621. MR 93i:17017.

[23] P.-O. Löwdin, Angular momentum wavefunctions constructed by projector operators, Rev. Modern Phys. 36 (1964), 966-976. MR 32\#8871.

[24] S. Majid, Beyond supersymmetry and quantum symmetry (an introduction to braidedgroups and braided-matrices), Quantum Groups, Integrable Statistical Models and Knot Theory (Tianjin, 1992), World Scientific Publishing, New Jersey, 1993, pp. 231282. MR 96h:81031.

[25] Y. I. Manin, Quantum Groups and Noncommutative Geometry, Université de Montréal Centre de Recherches Mathématiques, Quebec, 1988. MR 91e:17001.

[26] P. J. Olver and J. A. Sanders, Transvectants, modular forms, and the Heisenberg algebra, Adv. in Appl. Math. 25 (2000), no. 3, 252-283. MR 2001j:11016.

[27] M. Rahman, Discrete orthogonal systems corresponding to Dirichlet distribution, Utilitas Math. 20 (1981), 261-272. MR 83c:33006. Zbl 0477.33007.

[28] _ Reproducing kernels and bilinear sums for q-Racah and q-Wilson polynomials, Trans. Amer. Math. Soc. 273 (1982), no. 2, 483-508. MR 83k:33023. Zbl 0492.33011.

[29] H. Rosengren, Multilinear Hankel forms of higher order and orthogonal polynomials, Math. Scand. 82 (1998), no. 1, 53-88. MR 99m:47033. Zbl 0946.33012.

[30] - On the triple sum formula for Wigner $9 j$-symbols, J. Math. Phys. 39 (1998), no. 12, 6730-6744. MR 99k:81084. Zbl 0935.81036.

[31] __ Multivariable orthogonal polynomials and coupling coefficients for discrete series representations, SIAM J. Math. Anal. 30 (1999), no. 2, 232-272 (electronic). MR 2001f:33015. Zbl 0946.33013.

[32] __ Multivariable orthogonal polynomials as coupling coefficients for Lie and quantum algebra representations, Ph.D. thesis, Lund University, 1999.

[33] _ A non-commutative binomial formula, J. Geom. Phys. 32 (2000), no. 4, 349-363. MR 2001b:33025. Zbl 01422967.

[34] Y. Shibukawa, Clebsch-Gordan coefficients for $U_{\mathcal{q}}(\mathrm{su}(1,1))$ and $U_{\mathcal{q}}(\mathrm{sl}(2))$, and linearization formula of matrix elements, Publ. Res. Inst. Math. Sci. 28 (1992), no. 5, 775-807. MR 93k:17036. Zbl 0801.17015.

[35] Y. F. Smirnov, V. N. Tolstoy, and Y. I. Kharitonov, Projection operator method and q-analog of angular momentum theory, Symmetries in Science, V (Lochau, 1990), Plenum, New York, 1991, pp. 487-518. MR 92j:81107.

[36] H. M. Srivastava, Certain q-polynomial expansions for functions of several variables, IMA J. Appl. Math. 30 (1983), no. 3, 315-323. MR 85d:33007.

[37] V. N. Tolstoy, Extremal projectors for quantized Kac-Moody superalgebras and some of their applications, Quantum Groups (Clausthal, 1989), Lecture Notes in Phys., vol. 370, Springer, Berlin, 1990, pp. 118-125. MR 93h:17046. Zbl 0726.17033.

[38] M. V. Tratnik, Multivariable biorthogonal Hahn polynomials, J. Math. Phys. 30 (1989), no. 3, 627-634. MR 90i:33025. Zbl 0661.33010. 
[39] L. L. Vaksman, q-analogues of Clebsch-Gordan coefficients, and the algebra of functions on the quantum group SU(2), Dokl. Akad. Nauk SSSR 306 (1989), no. 2, 269-271. MR 90k:33018. Zbl 0693.33006.

[40] A. Verma, Some transformations of series with arbitrary terms, Ist. Lombardo Accad. Sci. Lett. Rend. A 106 (1972), 342-353. MR 48\#6486. Zbl 0263.33003.

[41] N. J. Vilenkin and A. U. Klimyk, Representation of Lie Groups and Special Functions, vol. 1, Mathematics and its Applications (Soviet Series), vol. 72, Kluwer Academic Publishers, Dordrecht, 1991. MR 93h:33009.

[42] Y. Xu, Summability of Fourier orthogonal series for Jacobi weight functions on the simplex in $\mathbf{R}^{d}$, Proc. Amer. Math. Soc. 126 (1998), no. 10, 3027-3036. MR 99i:42039. Zbl 0918.42015.

[43] D. Zagier, Introduction to modular forms, From Number Theory to Physics (Les Houches, 1989), Springer, Berlin, 1992, pp. 238-291. MR 94e:11039. Zbl 0791.11022.

[44] A. S. Zhedanov, $q$ rotations and other $q$ transformations as unitary nonlinear automorphisms of quantum algebras, J. Math. Phys. 34 (1993), no. 6, 2631-2647. MR 94g:17035. Zbl 0789.17011.

HJalmar Rosengren: Department of MATHEMATics, Chalmers UNIVERSity OF TeCHNOLOGY AND GÖTEBORG UNIVERSITY, SE-412 96, GÖTEBORG, SWEDEN

E-mail address: hja7mar@math.cha7mers.se 


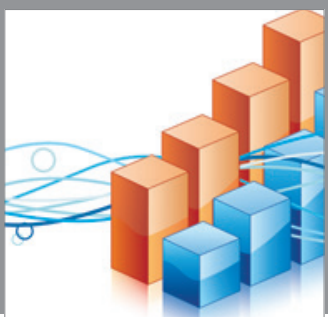

Advances in

Operations Research

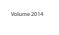

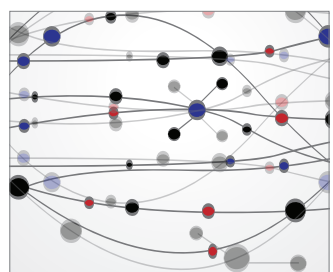

\section{The Scientific} World Journal
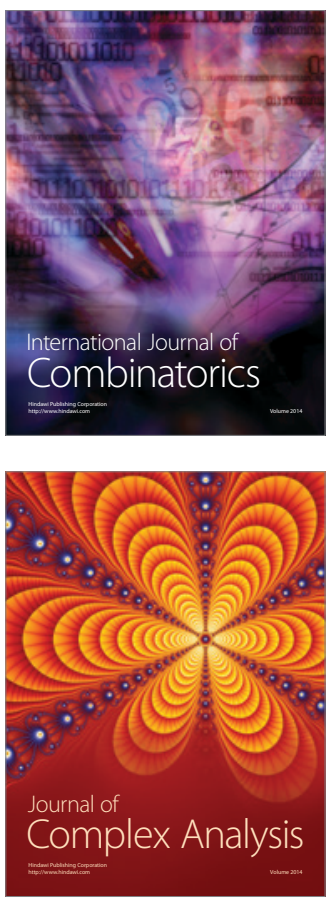

International Journal of

Mathematics and

Mathematical

Sciences
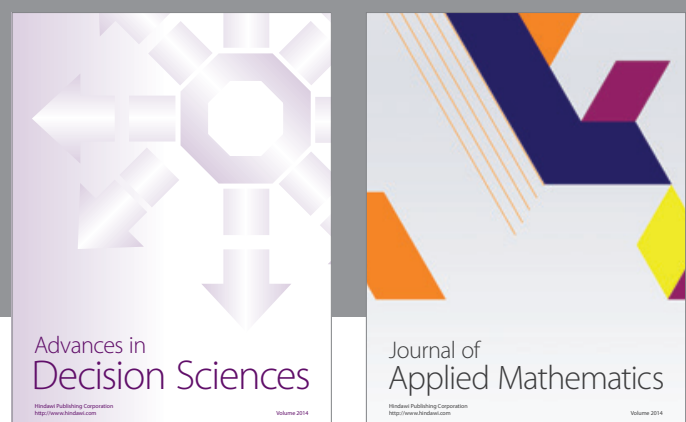

Journal of

Applied Mathematics
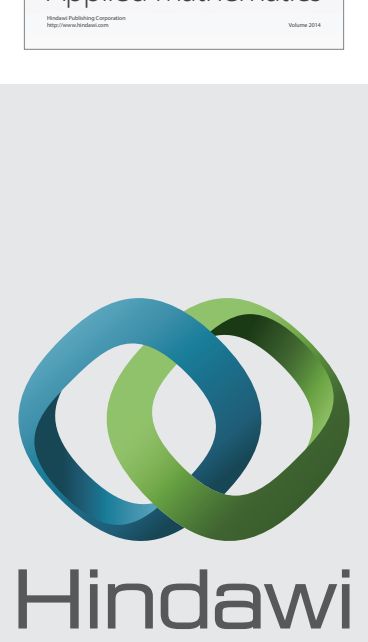

Submit your manuscripts at http://www.hindawi.com
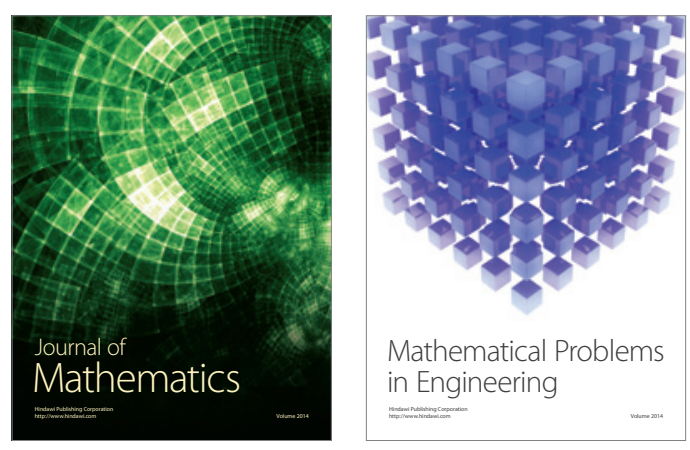

Mathematical Problems in Engineering
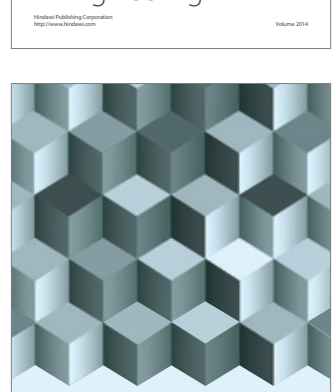

Journal of

Function Spaces
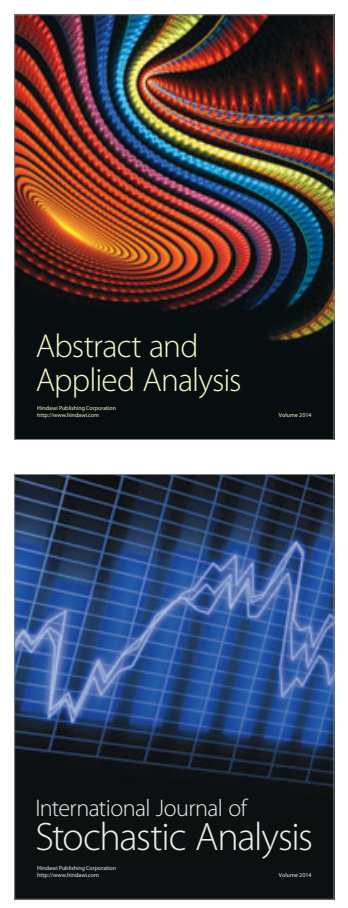

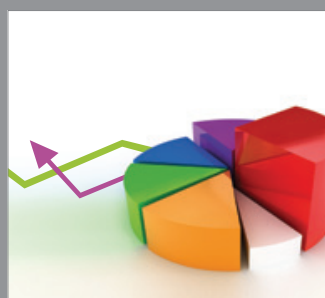

ournal of

Probability and Statistics

Promensencen
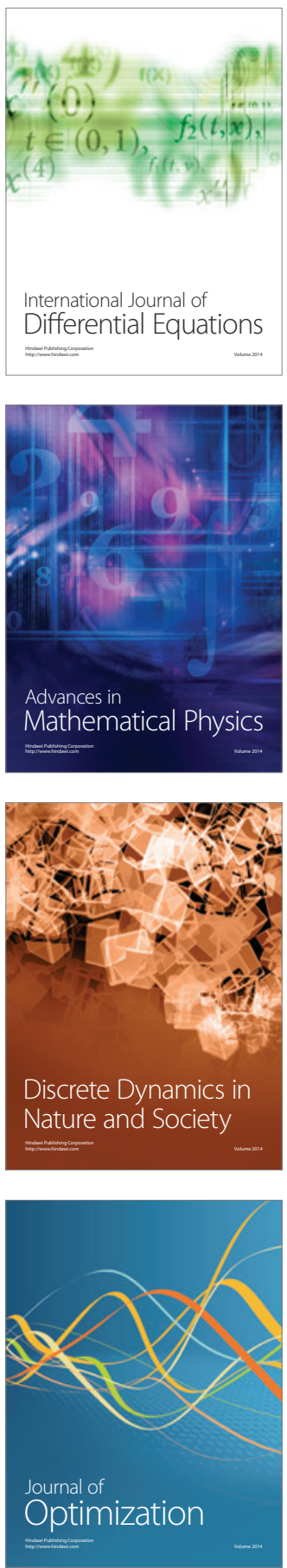